\title{
Carbon Deflagration in Type Ia Supernova: I. Centrally Ignited Models
}

\author{
H. Ma ${ }^{1}$, S. E. Woosley ${ }^{1}$, C. M. Malone ${ }^{1}$, A. Almgren ${ }^{2}$, and J. Bell ${ }^{2}$
}

\begin{abstract}
A leading model for Type Ia supernovae (SNe Ia) begins with a white dwarf near the Chandrasekhar mass that ignites a degenerate thermonuclear runaway close to its center and explodes. In a series of papers, we shall explore the consequences of ignition at several locations within such dwarfs. Here we assume central ignition, which has been explored before, however, the problem is worth revisiting, if only to validate those previous studies and to further elucidate the relevant physics for future work. A perturbed sphere of hot iron ash with a radius of $\sim 100 \mathrm{~km}$ is initialized at the middle of the star. The subsequent explosion is followed in several simulations using a thickened flame model in which the flame speed is either fixed — within the range expected from turbulent combustion or based on the local turbulent intensity. Global results, including the explosion energy and bulk nucleosynthesis (e.g. ${ }^{56} \mathrm{Ni}$ of $0.48-0.56 \mathrm{M}_{\odot}$ ) turn out to be insensitive to this speed. In all completed runs, the energy released by the nuclear burning is adequate to unbind the star, but not enough to give the energy and brightness of typical SNe Ia. As found previously, the chemical stratification observed in typical events is not reproduced. These models produce a large amount of unburned carbon and oxygen in central low velocity regions, which is inconsistent with spectroscopic observations, and the intermediate mass elements and iron group elements are strongly mixed during the explosion.
\end{abstract}

Subject headings: supernovae: general - Type Ia - stars: - hydrodynamics

\section{INTRODUCTION}

Common Type Ia supernova (SN Ia) are believed to be the thermonuclear explosions of carbon-oxygen white dwarfs in binary systems (Hoyle \& Fowler 1960; Hillebrandt \& Niemeyer

\footnotetext{
${ }^{1}$ Department of Astronomy and Astrophysics, University of California, Santa Cruz, CA 95064

${ }^{2}$ Center for Computational Sciences and Engineering, Lawrence Berkeley National Lab, Berkeley, CA 94720
} 
2000). Beyond this generality though lies room for considerable diversity. The white dwarf can grow to criticality by accreting mass slowly from a companion star, the so called "singledegenerate" model, or by merging with another white dwarf, the "double degenerate model." Depending upon the accretion rate and angular momentum transfer, the mass of the white dwarf when the nuclear runaway begins can vary upwards (Howell et al. 2006) or downwards (e.g. Woosley \& Kasen 2011) from the Chandrasekhar value (1.38 $\mathrm{M}_{\odot}$ for ignition). Ignition itself may happen in the center, slightly off-center, or near the surface of the white dwarf. Historically, greatest attention has been given to the centrally ignited Chandrasekhar mass model (the MCh model). This focus partially reflected the predominance, until recently, of one-dimensional (hence spherically-symmetric) codes and models, but also the success of the model itself. Given the liberty of varying uncertain flame physics, including the possibility of a delayed detonation, the MCh model has demonstrated striking agreement with the observed energetics, light curves, spectra, and nucleosynthesis of common SN Ia events (Nomoto, Thielemann \& Yokoi 1984; Branch et al. 1985; Höflich \& Khokhlov 1996; Mazzali et al. 2007).

More recently though, it has been realized that other models are probably needed, especially if one is to explain unusual events (e.g. Howell et al. 2006; Stritzinger et al. 2006; Perets et al. 2010; Pakmor et al. 2011). Simulations with increased complexity and realism have also shown that the MCh model itself probably doesn't ignite exactly in its center Garcia-Senz \& Wooslev 1995; Höflich \& Stein 2002; Kuhlen, Woosley \& Glatzmaier 2006; Zingale et al. 2011; Nonaka et al. 2012). This symmetry breaking may have important observational consequences for the explosion mechanism (Plewa et al. 2004; Röpke et al. 2007b), as well as the spectrum and light curve (Kasen et al. 2009). Still it remains interesting to explore the traditional model. Despite x-ray limits on the progenitor system (Gilfanov \& Bogdán 2010), the single-degenerate model remains viable (Voss \& Nelemans 2008; Hachisu et al. 2010), and even in double degenerate systems, the mergers of white dwarf pairs may produce MCh models as well as super-MCh models (Yoon et al. 2007). Studies of off-center ignition also show a small, but non-negligible probability of ignition occurring so close to the center that it probably burns through to the other side (Zingale \& Dursi 2007; Nonaka et al. 2012).

In the last decade, the symmetric MCh model has also attracted considerable attention from the three-dimensional simulators. Despite some initial optimism (Reinecke. Hillebrandt \& Niemeyer 2002; Röpke et al. 2006), these calculations (Gamezo et al. 2003; García-Senz \& Bravo 2005; Schmidt \& Niemeyer 2006; Röpke et al. 2007a) have shown that even though pure deflagration, i.e., deflagration without a subsequent detonation, released enough energy to unbind the star, it is unable to provide the larger kinetic energies characteristic of common SN Ia. Equally problematic, the deflagration-only models produce too little ${ }^{56} \mathrm{Ni}$, leave behind 
significant unburned carbon and oxygen with low speed near the center of the ejecta, and lack intermediate mass elements at high velocity. These aspects make it difficult for pure deflagration alone to produce a common SN Ia consistent with observations, although see Kromer et al. (2013) for pure deflagration models of sub-luminous SNe Ia.

Here we revisit this classic problem of centrally ignited deflagration in MCh models, not with the intention to prove that they work, but to understand better why they don't work. Our study has several motivations. First, different groups have assumed different initial conditions, codes, grid geometries, and resolutions. With the power of adaptive mesh refinement (AMR) and larger computers, we are able to define the initial conditions more precisely and track the burning more realistically. Previous studies also disagree concerning how much carbon is left at the center after the explosion is over and on the amount of intermediate mass elements (IME) produced. We want to understand this and give an alternate set of results. We are also implementing a novel, inexpensive approach to flame propagation that uses tables of yields generated off-line assuming isobaric burning and want to test the sensitivity of results to uncertain parameters in a well-studied case. Finally, in order to study delayed detonation in the MCh model as we plan to do in a subsequent paper, it is necessary to better understand the conditions that exist at the transition.

In $\S 2$ we briefly describe the fully compressible hydrodynamics code, CASTRO used in these studies (see also Almgren et al.2010), and describe our treatment of the flame physics. Details of the calculations are presented in $\S$, followed by the results of our simulations in $\S$ 4. Discussion and comparison to previous work are presented in $\S 5$.

\section{The CASTRO Code and Numerical Implementation of the Problem}

\subsection{CASTRO}

Because numerous instabilities are involved in the propagation of the burning and the important role played by turbulence, realistic studies must be carried out in three dimensions. To avoid any grid singularities, it is also helpful if the mesh is Cartesian. Coupled with the requirement that the burning regions themselves be well resolved implies that this is a supercomputer problem. Even with today's largest machines, a calculation that resolves the real flame surface $\left(\sim 10^{-2} \mathrm{~cm}\right)$ and covers the full star $\left(\sim 10^{8} \mathrm{~cm}\right)$ over the duration of the supernova explosion is impossible. Instead we rely on implicit large eddy simulations with adaptive mesh refinement (AMR) to provide the highest (feasible) resolution only in the regions where it is most needed.

Over the last five years, our group has developed a code that specifically fits these re- 
quirements. CASTRO (Almgren et al. 2010) is a fully compressible, finite-volume radiationhydrodynamics code that uses an unsplit version of the piecewise parabolic method (PPM). The grid is Eulerian and uses a hierarchical block-structured approach to AMR. CASTRO does not require a strict parent-child relation between patches in different levels (Figure 1 in Almgren et al. (2010) ), and performs sub-cycling in time-step advance for the refined region. Both features enhance its efficiency on massively parallel machines.

Self-gravity is supported in CASTRO using a Poisson solver, as well as an economic monopole approximation, which creates a 1D radial gravity field consistent with the mass distribution. The latter was used here. Users may supply external modules to define a suitable equation of state (EOS) and nuclear reaction networks. The user can control the regions that are refined based upon combinations of many physical parameters, or their gradients. The mesh spacing can vary by a factor of 2 or 4 between levels. Time steps at each level are constrained by a standard CFL condition based on maximum wave speed over the grids at that level. Additional limitations can be imposed by the user to follow the temporal evolution of composition changes by nuclear reactions. Another useful feature is the ability to enlarge the computational domain from a restart file. In our simulation, the final domain is 32 times larger than the original domain. Thus while the grid is not co-moving with the matter, it can follow explosions over a large increase in the initial radius to obtain a homologous coasting configuration. Modules can also be provided to describe the initial model and flame propagation.

\subsection{Mapping and Initialization}

The presupernova model employed here is a $1.38 \mathrm{M}_{\odot}$ white dwarf with an initial composition of $50 \%$ carbon and $50 \%$ oxygen, generated using KEPLER, a 1D implicit Lagrangian code (Weaver, Woosley \& Zimmerman 1978; Woosley et al. 2002). This white dwarf model has been previously used many times as a starting point for studies of SN Ia (e.g. Niemeyer \& Wooslev 1997; Wooslev et al. 2007) and is similar to the initial model of Nomoto's Model "W7" (Nomoto, Thielemann \& Yokoi 1984). The central density of the star at the time of the final runaway was $2.6 \times 10^{9} \mathrm{~g} \mathrm{~cm}^{-3}$, the central temperature, $6 \times 10^{8} \mathrm{~K}$, and the initial radius, about $1700 \mathrm{~km}$. To ensure hydrostatic equilibrium on the new Cartesian grid, the distribution of physical parameters was adjusted from their values in KEPLER to satisfy the differencing formulae, $0.5\left(\rho_{i}+\rho_{i-1}\right) g=\left(P_{i}-P_{i-1}\right) / d r$. Constant entropy was assumed in the convection zone which includes most of the mass of the presupernova star. Here $g=G M(r) / r^{2}$ was defined on the interface between zones, where $M(r)$ is the enclosed mass at the radius of $r$. For the multi-dimensional calculations, a very fine grid was first gen- 
erated in hydrostatic equilibrium in the 1D version of CASTRO using spherical coordinates. This grid was then interpolated onto the Cartesian grid AMR hierarchy to give the starting model in 3D. The Helmholtz stellar equation of state (EOS) of Frank Timmes was used in all of our calculations (Timmes \& Swesty 2000). Because the Eulerian grid requires non-zero values in all zones, the white dwarf was surrounded by an artificial, but inert, low-density medium with temperature $5 \times 10^{6} \mathrm{k}$ and density $10^{-4} \mathrm{~g} \mathrm{~cm}^{-3}$. This low density medium had no dynamic effect on the supernova explosion calculation. A numerical "sponge" was implemented to damp the velocity in the surrounding medium where densities were between $10^{-4}$ and $1000 \mathrm{~g} \mathrm{~cm}^{-3}$. This was done by multiplying the three velocity components by a factor that varied smoothly from nearly 0 to 1 in this density range (Zingale et al. 2009). The sole purpose of the sponge was to keep the time step in the low density, artificial circumstellar medium from affecting the calculation as would happen if the medium began to accrete onto the white dwarf.

Burning was initiated by a nearly spherical bubble of hot iron ash surrounding the center of the star with a radius of $100 \mathrm{~km}$. The temperature in the ash was set to 8.5 billion $\mathrm{K}$, and binding energy per nucleon, $B E$, and mean atomic weight, $\bar{A}$, were set using the nuclear burning tables (Tables 1 and 2) to $8.17 \mathrm{MeV} /$ nucleon and 11.6 respectively. The ash density was derived from the equation of state using this temperature and the same pressure as the surrounding fuel. Since the initial ash was in nuclear statistical equilibrium (NSE), its abundances and $Y_{e}$ started to evolve immediately. The surface of this sphere was perturbed using spherical harmonic functions assuming a superposition of all $l$-wave modes from 14 to 20 and a maximum perturbation amplitude of about $25 \mathrm{~km}$. The choice of $100 \mathrm{~km}$ for the radius was influenced by studies of ignition (e.g. Nonaka et al. 2012) which showed that prompt ignition outside this radius was very unlikely. This is a smaller initial radius than has been employed by some groups (Röpke et al. 2006, 2007a). The initial zoning and AMR structure employed are described in $\S$ 3.1. Pressure was kept unchanged across the ash fuel interface, though the ash had lower density and higher temperature. The initial perturbed sphere was therefore Rayleigh-Taylor unstable, though it took some time for these initial instabilities to grow. This use of a single large deformed ignition region seems more physical

to us than ignition at many numerous distinct points (Röpke et al. 2006), especially since recent studies show that the flame only ignites once (Nonaka et al. 2012).

\subsection{Nuclear Physics of the Burning}

The nuclear physics of high temperature carbon deflagration can be quite complex. The products of the burning depend upon the density and may or may not be in NSE. After the 
deflagration front has passed, the composition continues to evolve through electron capture and the reassembly of partially photodisintegrated matter. At low density, nuclear burning can continue. Electron capture changes the electron mole number, $Y_{e}$, to which the pressure is quite sensitive. Reassembly of alpha-particles into iron-group elements provides a substantial energy boost as the composition cools by expansion. Nuclear fusion can appreciably alter the final composition, especially at lower temperatures where NSE is not achieved. These effects can only be followed accurately with a a large nuclear reaction network. Yet at the same time, coupling a large network directly to a 3D simulation would be very computationally expensive. Worse still, the nuclear time step can be very small, especially in NSE, and the solution to the stiff system of ODEs is prone to numerical instability.

Our solution was to use tables to describe the energy generation and composition changes. The tables were generated off-line using a large nuclear reaction network whose accuracy has been tested many times in the Kepler stellar evolution code. Different tables were used to describe the burning in the flame $\S 2.3 .1$ and the volumetric burning in NSE $\S$ 2.3.2. The implementation of this burning in a flame model is discussed in $\S 2.4$.

\subsubsection{Burning in the Flame}

The first stage of the burning occurs as the flame passes through the unburned carbon and oxygen. At the high density of the deep white dwarf interior, this flame is very thin, though highly convoluted, essentially a moving unresolved discontinuity that turns carbon and oxygen into iron-group elements, alpha-particles, and nucleons in NSE. At lower densities, the heat capacity of the matter rises and the burning results in a lower temperature. Carbon, oxygen, neon and silicon burning regions in the flame grow thicker and eventually burning no longer culminates in NSE, but in the production of intermediate mass elements, silicon through calcium. At still lower density, the oxygen does not burn to completion and one has neon, oxygen and magnesium. Eventually the burning goes out.

A characteristic shared at all densities though is that the burning in the flame transpires at constant pressure. Detonation does not occur in the models being studied, and because the flame speed during the deflagration stage is such a small fraction of the sound speed $(\sim 1 \%)$, pressure equilibrates across the flame. Since the gas is initially very degenerate and has $Y_{e}=0.50$, the pressure in the fuel, and hence in the ash, is completely determined by the fuel's density and is not very sensitive to its (low) temperature. Assuming a reasonable limit to the time scale for the burning to continue once the energy generation saturates at a maximum, the change in composition at a given density can be uniquely determined from the initial composition and the isobaric condition. This allows the energy yield and ash 
composition to be computed off-line using a reaction network of arbitrary size.

Beginning at a low temperature, taken here to be $6 \times 10^{8} \mathrm{~K}$, and for a grid of initial densities from $10^{6.4}$ to $10^{10.0} \mathrm{~g} \mathrm{~cm}^{-3}$ in steps of $\log \rho$ of 0.05 , the results of nuclear burning were calculated using a nuclear reaction network that contained 188 isotopes, including neutron, proton, ${ }^{4} \mathrm{He},{ }^{12-14} \mathrm{C},{ }^{14-15} \mathrm{~N},{ }^{16-18} \mathrm{O},{ }^{18-19} \mathrm{~F},{ }^{19-22} \mathrm{Ne},{ }^{22-23} \mathrm{Na},{ }^{23-28} \mathrm{Mg},{ }^{26-29} \mathrm{Al}$, ${ }^{27-32} \mathrm{Si},{ }^{31-36} \mathrm{P},{ }^{30-33} \mathrm{~S},{ }^{34-39} \mathrm{Cl},{ }^{35-42} \mathrm{Ar},{ }^{38-42} \mathrm{~K},{ }^{39-48} \mathrm{Ca},{ }^{41-49} \mathrm{Sc},{ }^{43-52} \mathrm{Ti},{ }^{45-53} \mathrm{~V},{ }^{47-56} \mathrm{Cr}$, ${ }^{49-57} \mathrm{Mn},{ }^{51-60} \mathrm{Fe},{ }^{53-61} \mathrm{Co},{ }^{55-64} \mathrm{Ni},{ }^{57-65} \mathrm{Cu},{ }^{59-68} \mathrm{Zn},{ }^{61-69} \mathrm{Ga}$ and ${ }^{63-72} \mathrm{Ge}$. The initial composition was $50 \%{ }^{12} \mathrm{C}$ and $50 \%{ }^{16} \mathrm{O}$ by mass. Burning proceeded with an imposed isobaric condition. That is, as time passed the temperature rose and density fell in response to the energy generation, but the changes in each were such as to keep the pressure constant for the appropriate equation of state. The "Helmholtz" equation of state was used to obtain the pressure, its derivatives, and the heat capacity at constant pressure during this iteration (Timmes \& Swesty 2000).

For each point in the $\rho-T$ grid, after a lengthy time, which would be much shorter in the star due to conduction, the temperature gradually began to rise and the rise accelerated along with the nuclear energy generation. For each density, there came a point where the nuclear energy generation reached a maximum and then began to decline due to the depletion of the most incendiary parts of the composition. Once the energy generation had declined to $80 \%$ of that maximum value, a counter was set and $0.1 \mathrm{~s}$ later the calculation was halted and the characteristics of the ash sampled. The $0.1 \mathrm{~s}$ is arbitrary, but the composition and temperature were not changing much during this time. The value chosen is a lower bound to the hydrodynamic expansion time of the star and an upper bound to the time for burning to cross the typical five zone thickness of the (thickened) flame.

This procedure is an approximation to what would actually happen in a multi-zone calculation of a steady state laminar flame. In a flame, conduction, not gradual nuclear burning, would heat the material to the point that it flashed on a time scale sufficiently short to sustain a self-similar front with a thickness given by the Landau condition (Landau \& Lifshitz 1959), which says that the nuclear time balances the energy diffusion time. Later in the burning, a small amount of heat would diffuse out of the ash into the fuel in order to ignite it and keep the flame going. However, because of the extreme temperature sensitivity of the burning rates and the small heat capacity of the degenerate matter, the amount of heat that must diffuse to propagate the flame is small. The major part of the burning thus occurs in thermal isolation at constant pressure and can be approximated by a single zone as we have done here. It is necessary only to resolve the energy generation spike that is most sensitive to the density of the fuel, and the small amount of burning that occurs after that spike. If the flame were very much broader or slower moving and the star did not expand so that 
the $0.1 \mathrm{~s}$ time scale was orders of magnitude longer, the ashes would be different, but the necessary change in time scale to have a major effect is very large. In the future, if flames need to be studied in very different circumstances, it would not be difficult to couple the off-line reaction network to a multi-zone (1D) flame model (Woosley et al. 2009).

The validity of the approximation is demonstrated by the good agreement with previous fine-zoned studies of laminar flames under the same conditions. Table 3 shows the comparison with other studies that also used either a $50 \%$ C, $50 \%$ O composition (Timmes \& Wooslev 1992; Woosley 2007) or a 40\% C, 60\% O composition (Woosley et al. 2011, indicated with an asterisk). Overall the agreement between ash temperature and density changes found in these studies is excellent. For the limited compositional information given in these references, the agreement was also very good.

The resulting composition from our network simulations was packed into the seven abundance variables carried by the $3 \mathrm{D}$ code depending on the atomic weight of the product nucleus. Species lighter than $\mathrm{A}=12$ were packed into helium; species from 12 through 15 into carbon; 16 through 19 into oxygen; 20 through 23 into neon; 24 through 27 into magnesium; and 28 through 47 into silicon. Hence all intermediate mass elements, silicon through calcium are contained in the "silicon" variable. Species heavier than A $=47$ were generically considered to be "iron" (Table 1). Additionally the final temperature, density, binding energy per nucleon, and mean atomic weight, $\bar{A}$, were determined for the ash (Table 2).

Once the change in energy and composition across the flame is known as a function of the initial density, the burning can be implemented without any recourse to temperaturedependent reaction rates or network solution. The flame is essentially a thin phase transition moving with a desired speed. The interface can be treated either using a level-set approach, or as was done here, as a thickened flame. The progress variable is the carbon mass fraction. As its mass fraction varies from 0.5 to zero, the binding energy per nucleon changes linearly from its value in the fuel, $7.828 \mathrm{MeV}$ per nucleon, to its value given for carbon-free ash in the tables. The seven abundance variables are interpolated linearly from their values in the fuel (zero for all but $\mathrm{C}$ and $\mathrm{O}$ ) and in the ash. The nuclear energy generation rate was

$$
\epsilon_{\mathrm{nuc}}=9.64 \times 10^{17}(\Delta B E / 0.5)\left(d X\left({ }^{12} C\right) / d t\right) \operatorname{erg~g}^{-1} \mathrm{~s}^{-1}
$$

Here 0.5 is the initial mass fraction of carbon and $\triangle B E$ is the change in binding energy per nucleon following the burning of a composition of $50 \% \mathrm{C}$ and $50 \% \mathrm{O}$ to ash (Table 2). Though the oxygen usually burns as well, carbon was the progress variable. The change in the carbon abundance itself was calculated using a thickened flame model ( $\$$ 2.4 $)$. Several one dimensional studies were carried out with the CASTRO code that showed this prescription 
for energy generation and composition change gave the same resultant temperatures and densities for a conductive flame as in the one zone network simulation.

For fuel densities below $10^{6.5} \mathrm{~g} \mathrm{~cm}^{-3}$ it was assumed that no nuclear burning took place and no burning was allowed on the grid until conduction had raised the local temperature in a carbon-rich composition above $2 \times 10^{9} \mathrm{~K}$.

\subsubsection{Volumetric Burning in Nuclear Statistical Equilibrium}

In NSE, all strong and electromagnetic nuclear reactions have come into a state of near balance with their reverse reactions, e.g. $(\mathrm{n}, \gamma),(\mathrm{p}, \gamma)$, and $(\alpha, \gamma)$ reactions are balanced by $(\gamma, \mathrm{n}),(\gamma, \mathrm{p})$, and $(\gamma, \alpha)$ reactions. The distribution of abundances is then completely specified by just three parameters: the temperature, density and the neutron excess. The latter is usually measured by the electron mole number,

$$
Y_{e}=\Sigma Z_{i} Y_{i}
$$

where $Y_{i}$ is the mass fraction of the species "i" divided by its atomic weight and $Z_{i}$ is its nuclear charge. For the initial composition and for the ash immediately behind the flame, there is neutron-proton equality so that $Y_{e}=0.50$.

The NSE composition can evolve, however, both as a consequence of the changing temperature and density and the decrease of $Y_{e}$ due to electron capture on nuclei at high densities $\rho \gtrsim 10^{8} \mathrm{~g} \mathrm{~cm}^{-3}$. Of particular importance is the fact that, at the high temperatures appropriate to the central regions of exploding white dwarfs, NSE favors the partial photodisintegration of iron group nuclei into alpha-particles. This tends to reduce the initial increase in nuclear binding energy and decrease the average atomic mass of the ions. The temperature of the ash is not as high as one would obtain for fusion just to iron. However, as the matter expands and cools the alpha-particles reassemble, providing late time energy input to the explosion. Also important is the reduction of $Y_{e}$ by electron capture. Since relativistic electrons are providing the pressure and since that pressure depends on the combination $\left(\rho Y_{e}\right)^{4 / 3}$, this can affect the dynamics. At constant pressure, a decrease in $Y_{e}$ means an increase in the density and a reduction in buoyancy so plumes rise less rapidly. The value of $Y_{e}$ also affects the nucleosynthesis and light curve of the supernova. For values close to 0.50 , the principle nucleosynthetic product is radioactive ${ }^{56} \mathrm{Ni}$, but for $Y_{e} \lesssim 0.48,{ }^{54} \mathrm{Fe}$ and ${ }^{58} \mathrm{Ni}$ are more abundant.

The evolution of the NSE composition can only be followed using a large network of nuclei since many nuclei participate in capturing electrons at different densities and many reactions are involved in maintaining the equilibrium. We used the 127 isotope equilibrium 
network from the Kepler stellar evolution code (Weaver, Woosley \& Zimmerman 1978) with weak interaction rates taken from a variety of sources as discussed in Heger et al. (2001). Particularly important are the revisions to earlier rates by Langanke \& Martinez-Pinedo (2000) and the inclusion of accurate rates for beta-decay and positron emission as well as electron capture. Temperature dependent partition functions were used in calculating the abundances. The isotopes included in the NSE network were neutrons, ${ }^{1-3} \mathrm{H},{ }^{3-5} \mathrm{He},{ }^{5} \mathrm{Li}$, ${ }^{9} \mathrm{Be},{ }^{12} \mathrm{C},{ }^{16} \mathrm{O},{ }^{20} \mathrm{Ne},{ }^{23} \mathrm{Na},{ }^{24-26} \mathrm{Mg},{ }^{26-28} \mathrm{Al},{ }^{28-30} \mathrm{Si},{ }^{30-33} \mathrm{P},{ }^{31-34} \mathrm{~S},{ }^{35-37} \mathrm{Cl},{ }^{36-40} \mathrm{Ar},{ }^{39-43} \mathrm{~K}$, ${ }^{40-48} \mathrm{Ca},{ }^{44-49} \mathrm{Sc},{ }^{44-52} \mathrm{Ti},{ }^{47-54} \mathrm{~V},{ }^{48-56} \mathrm{Cr},{ }^{51-58} \mathrm{Mn},{ }^{52-62} \mathrm{Fe},{ }^{54-64} \mathrm{Co},{ }^{56-66} \mathrm{Ni},{ }^{59} \mathrm{Cu}$, and ${ }^{60} \mathrm{Zn}$.

The table was constructed to include, as a function of the three independent variables, $\rho$, T, and $Y_{e}$, the binding energy per nucleon of the NSE distribution, its average atomic weight, $\bar{A}$, the helium mass fraction, and the time rate of change of $Y_{e}$ resulting from all the weak interactions. Thirty one values of density from $10^{7}$ to $10^{10} \mathrm{~g} \mathrm{~cm}^{-3}, 71$ temperatures from $10^{9}$ to $10^{10.4} \mathrm{~K}$, and 21 values of $Y_{e}$ from 0.50 to 0.40 were included in the table (46221 grid points). The temperature and density grid was in equal steps of the base $10 \mathrm{log}$. The values of $\Delta Y_{e}$ were also equal so table look up time was minimized. Linear interpolation in $\log \mathrm{T}, \log \rho$ and $Y_{e}$ thus gave the binding energy, mean atomic mass, and time rate of change of $Y_{e}$ for any point of interest in the calculation. NSE was only assumed to exist in a zone if its density was over $10^{8} \mathrm{~g} \mathrm{~cm}^{-3}$, its temperature above $3 \times 10^{9} \mathrm{~K}$, its carbon mass fraction was below $1 \%$, and the combined mass fractions of iron and helium were over $88 \%$.

Due to the stiff dependence of abundances and binding energy on temperature and density, NSE calculations are subject to a well known instability. If the temperature goes up at constant density, for example, photodisintegration can give a large negative energy generation rate. That will tend to reduce the temperature for the next call which results in recombination and a large positive energy generation. Since the time scale for this adjustment is instantaneous, the code may take very small times steps and still have convergence problems. This difficulty was circumvented here by the addition of numerical inertia to the NSE calculation. The change in mean binding energy implied by a temperature-density change was not applied fully in a single time step but spread over three time steps. In practice, this kept the calculation stable and asymptotic values for $Y_{e}$ and the binding energy were attained much more quickly than the time scale for the temperature or density to vary appreciably.

A few sample points from the NSE table are given in Table 4. In the actual table, six figure precision was maintained. The NSE network was not large enough, nor reliable weak interaction information available for the accurate calculation of abundances and $\dot{Y}_{e}$ for $Y_{e}$ below 0.43 . Fortunately, the smallest value of $Y_{e}$ in any of our simulations here was above 0.45 . 


\subsection{Flame Model}

Burning during the deflagration stage of a SN Ia is quite complex and has challenged model builders for years. The most interesting burning during this stage happens at sufficiently low Karlovitz number, $K a \lesssim 1$, that the concept of a well defined laminar flame remains valid. At higher values of $K a$, which happen for densities below about $10^{7} \mathrm{~g} \mathrm{~cm}^{-3}$, turbulence may lead to mixing, at the microscopic level, of cold fuel and hot ash and a possible detonation (Niemeyer \& Woosley 1997; Khokhlov et al. 1997; Woosley et al. 2009). That possibility is ignored here. Thus the picture of the flame is of a surface deformed by floatation, various instabilities, and possibly turbulence. Large scale motions caused by buoyancy are captured on the grid, but the smaller scale wrinkling is not. The manner in which this unresolved burning is treated is usually called the "subgrid model" for burning. Following Damköhler (1940), it is often assumed that if the large scale motions governing the spread of the flame are sufficiently resolved, e.g., the integral scale of the turbulence in the vicinity of the flame is well resolved, small scale burning will adapt so as to "digest" any entrained material at the necessary rate.

Adapting tools developed by the chemical combustion community, two approaches have been used by astrophysicists. One approach uses a "level set" (Osher \& Sethian 1988; Reinecke et al. 1999) and a subgrid model for turbulence (Schmidt et al. 2006a, b). The ash-fuel interface, found by solving a "G-equation", is moved at a speed given by sampling the velocity field on the grid and estimating the turbulent intensity. Typical speeds are $\sim 100$ $\mathrm{km} \mathrm{s}^{-1}$, though a wide range of values is found (Röpke 2007). An alternate approach is the "thickened flame model" (Davies \& Taylor 1950; Sharp 1984), where an artificial conductivity and energy generation are employed to spread the flame over several zones and force it to move at a prescribed speed. Both the flame speed and width can be much greater than the corresponding laminar values. The width is determined by the need to resolve the flame while the speed is sometimes taken to be the value to "burn out" structure at the grid level. It is well known (e.g. Timmes \& Woosley 1992), that flame perturbations smaller than

$$
\lambda_{\text {min }} \approx 4 \pi V^{2}\left(g \frac{\delta \rho}{\rho}\right)^{-1}
$$

will be burned before they can grow. This is sometimes called the "fire-polishing length". Conversely if one sets $\lambda_{\text {min }}$ equal to the resolution, say a few zones, one can solve for a characteristic speed, V. Thus $V \sim f \sqrt{g_{\text {eff }} \Delta x}$ where $g_{\text {eff }}=g(\Delta \rho) / \rho, \Delta x$ is the grid resolution, and $\mathrm{f}<1$. This approach has been adopted by most groups studying the problem in the US (Khokhlov 1995, 1996; Gamezo et al. 2003; Townsley et al. 2007). For a resolution of several $\mathrm{km}$ and $g_{\text {eff }} \sim 10^{9} \mathrm{~cm} \mathrm{~s}^{-2}$, this prescription also gives a characteristic speed $\sim 100 \mathrm{~km} \mathrm{~s}^{-1}$.

While we see great merit in the level set approach and are working on developing our 
own, the current study used a thickened flame. In particular, the nuclear burning time scale and diffusion time scale are defined here as,

$$
\tau_{n u c}=\tau_{d i f f}=\frac{n \Delta x}{V},
$$

where $n$ is the desired number of grid zones across the flame surface and $\Delta x$ is the grid spacing, so that $n \Delta x$ is the desired flame width. $V$ is the desired flame speed. The nuclear energy generation rate and the conductivity are then derived from prescribed flame width and velocity.

In the flame, where the carbon abundance is larger than 1 percent and temperature is beyond 2 billion degrees, the carbon consumption rate is defined as,

$$
\frac{d X\left({ }^{12} C\right)}{d t}=-\frac{0.5}{\tau_{\text {nuc }}}
$$

where 0.5 is the initial carbon mass fraction. Additionally,

$$
\frac{d(B E)}{d X\left({ }^{12} C\right)}=-\frac{\Delta B E}{0.5}
$$

so that nuclear burning rate in Equation (1) can be derived.

For partially burned cells inside the flame, the current $B E$ and $\bar{A}$ are interpolated using the equations:

$$
\begin{gathered}
\frac{B E-B E_{f}}{X\left({ }^{12} C\right)-0.5}=-\frac{B E_{f}-B E_{i}}{0.5}, \\
\frac{\bar{A}-\bar{A}_{f}}{X\left({ }^{12} C\right)-0.5}=-\frac{\bar{A}_{f}-\bar{A}_{i}}{0.5} .
\end{gathered}
$$

The opacity is also defined using $\tau_{\text {diff }}$ from the thickened flame model as

$$
\kappa=\frac{c \tau_{\text {diff }}}{3 \rho \Delta^{2}}
$$

and conductive coefficient is,

$$
D=4 a c T^{3} / 3 \rho \kappa
$$

where $c$ is light speed, $\Delta$ is the flame thickness, and $a=7.5646 \times 10^{-15} \mathrm{erg} \mathrm{cm}^{-3} \mathrm{~K}^{-4}$ is radiation density constant. The conductivity is only taken to be non-zero in zones whose temperature is above 1 billion degree. This criterion allows us to distinguish the flame and post-flame region from material heated diffusively in front of the flame. Suppressing spurious burning in the diffusion front ahead of burning front is necessary to prevent an artificial "diamond" shape from appearing in the propagation of the flame. 
From a survey of one dimensional flames using the above prescription, we determined the multiplier, $f_{w}$ and $f_{v}$ (both of order unity), of the flame width and speed to get prescribed values at different densities (see Table 5). In the calculation, the left side is set to be hot ashes, and right side is fresh fuel (Figure 1). The boundary condition is impermeable at the right side, free flow at the left side. Flame speeds are measured with respect to the fuel (Figure 2).

\subsection{Choice of Flame Speed Parameter}

It thus remains only to pick the desired flame speed. As discussed in $\S$ 2.4. previous studies using a subgrid model to describe the turbulent flow (Reinecke et al. 1999; Reinecke, Hillebrandt \& Niemeyer 2002; Röpke \& Hillebrandt 2005) give a speed on the order of $100 \mathrm{~km} \mathrm{~s}^{-1}$. In particular, Röpke (2007) studied the probability density function (PDF) of turbulent velocity at a density interval of $1-3 \times 10^{7} \mathrm{~g} \mathrm{~cm}^{-3}$ in the deflagration phase. His plot of the PDF shows a turbulent velocity peaking approximately 100-300 $\mathrm{km} \mathrm{s}^{-1}$. Thickened flame models for typical values of resolution give a comparable, though somewhat smaller value for $\mathrm{V}$. The laminar speed, which sets a lower bound to the overall flame speed, is almost this large at the high density where burning begins. At a density of $2 \times 10^{9} \mathrm{~g} \mathrm{~cm}^{-3}$, the laminar speed is $76 \mathrm{~km} \mathrm{~s}^{-1}$ (Timmes \& Woosley 1992), though it declines rapidly for densities much less than $10^{9} \mathrm{~g} \mathrm{~cm}^{-3}$. By the time the density has declined though, floatation is dominating with typical speeds of thousands of $\mathrm{km} \mathrm{s}^{-1}$ and the turbulent estimate or the fire polishing estimate is valid.

This all suggests that a constant flame speed of $100 \mathrm{~km} \mathrm{~s}^{-1}$ is a good place to begin and this is the value used in our standard calculation. However, we also carried out other otherwise identical calculations using fixed $\mathrm{V}=50$ and $200 \mathrm{~km} \mathrm{~s}^{-1}$. The lower value here is actually a little less than the laminar speed for a composition of $50 \%$ carbon, $50 \%$ oxygen at a density of $2.4 \times 10^{9} \mathrm{~g} \mathrm{~cm}^{-3}$ where the laminar speed has a value of $89 \mathrm{~km}$ $\mathrm{s}^{-1}$ (Timmes \& Woosley 1992). However, this high density and laminar speed are only maintained a short time and, in the interest of maintaining a constant value of $\mathrm{V}$ throughout the run, the lower value of $50 \mathrm{~km} \mathrm{~s}^{-1}$ was used. Interestingly, had the carbon mass fraction been $20 \%, 50 \mathrm{~km} \mathrm{~s}^{-1}$ would have been the correct value even at this high density.

To test the sensitivity of the results to the local turbulence, we also performed a simulation where the flame speed depended quite simply on the local turbulent intensity. In particular, for a zone within the flame (as described by the nuclear burning regime discussed 
in $\S 2.3 .1$ ) the turbulent velocity fluctuations, $v_{\text {turb }}$, are calculated in the usual way:

$$
v_{\text {turb }}=\left(\overline{v^{2}}-\bar{v}^{2}\right)^{1 / 2},
$$

where the overbar denotes spatial averaging over a cube of $3^{3}$ zones centered about the zone in question. The value of $v_{\text {turb }}$ from (11) is then rescaled to the grid scale assuming Kolmogorov scaling. The local flame speed, $\mathrm{V}$, is then set to the maximum between the laminar speed and the local turbulent intensity. As we shall see the answer is gratifyingly insensitive to the choice of $\mathrm{V}$ - constant or set to the local turbulent intensity - within the limited range explored.

\section{Calculations and Analysis}

\subsection{Calculations}

The initial computational domain of the 3D calculations is $8192 \mathrm{~km}$ in each dimension with $512^{3}$ cells at base level so that the largest cells were $(16 \mathrm{~km})^{3}$. We then added 4 levels of refinement using AMR so that the resolution at the finest level was $(1 \mathrm{~km})^{3}$. The finest resolution was always used in the vicinity of the flame. Specifically, fine zoning was used in the vicinity of any transitions in carbon mass fraction from 0.50 to less than 0.49 . Higher resolution was also used in the highest density regions in order to accurately describe the nuclear evolution and floatation of the first ashes. The coarsest resolution was used in the near vacuum outside the exploding star. In particular, a region was tagged for refinement when $\rho \Delta x^{3}>2 \times 10^{25} \mathrm{~g}$, where $\Delta x$ is the cell size. In order to maintain appropriate resolution as the flame surface area increased rapidly, the finest zoning was coarsened twice, once when total number of cells at all levels was more than 1 billion at $0.63 \mathrm{~s}$, and again when there were 1.5 billion cells at $0.87 \mathrm{~s}$ for the standard run. The zoning at the finest level was never more than $4 \mathrm{~km}$ until the completion of intermediate mass element production when the supernova's radius was about $10,000 \mathrm{~km}$.

As noted previously, a constant flame speed independent of the grid scale was assumed for all (hereafter, model A series) but one simulation (hereafter, model BT) that used the local turbulent speed. Three different choices were used for constant flame speed in three independent runs: 50 (model A50), 100 (model A100) and $200 \mathrm{~km} \mathrm{~s}^{-1}$ (model A200). The entire domain was remapped twice for models A50 and A200 to a grid twice as large each time, by adding another coarser AMR level than the previous base level, when the outer

edge of the supernova expanded close to the grid boundary. For model A100, the domain was remapped 5 times in order to follow the expansion to the homologously expanding state. 
Thus our biggest domain size was 262,144 km, 32 times larger than the original domain. For the A50 and A200 runs, we only followed the explosion for $1.8 \mathrm{~s}$ until the nuclear burning had shut off due to expansion. The simulation for model BT used slightly different random initial spherical harmonic perturbations than the A series of models. To compare model BT with the constant flame speed runs, we performed an additional simulation with the same initial conditions as model BT, but with constant $\mathrm{V}=100 \mathrm{~km} \mathrm{~s}^{-1}$, hereafter model B100. That is, the only difference between models A100 and B100 are the initial random perturbations of the ash. Models BT and B100 were only evolved until the first buoyant plume reached the surface, and thus did not require remapping to a larger domain. All calculations were typically run on 6,144 or 12,288 processors for 2-3 Million CPU hours each on Jaguarpf/Titan at the OLCF and on Franklin and Hopper at NERSC.

\subsection{Parsing Iron}

Once the composition had ceased to evolve, the electron mole number, $Y_{e}$, and helium mass fraction were used to distinguish the dominant forms of iron $\left({ }^{54,56} \mathrm{Fe},{ }^{56,58} \mathrm{Ni}\right)$ produced in the explosion. Three cases are considered based on neutron excess $\eta=1-2 Y e$ (Hartmann, Woosley \& El Eid 1985),

$$
\eta=\sum \frac{\left(N_{i}-Z_{i}\right) X_{i}}{A_{i}}
$$

For the conditions studied here, most of the neutrons will be bound in ${ }^{54} \mathrm{Fe},{ }^{56} \mathrm{Fe}$, and ${ }^{58} \mathrm{Ni}$ with the relative proportions depending upon the values of $\eta$ and the helium mass fraction, $\mathrm{X}(\mathrm{He})$. For near proton-neutron equality, i.e., $\eta$ less than $1 / 27$, and $\mathrm{X}(\mathrm{He})$ less than 0.20 , it is assumed that most of the neutrons reside in ${ }^{54} \mathrm{Fe}$ with a small fraction in ${ }^{58} \mathrm{Ni}$. Specifically $\eta=1 / 27 \mathrm{X}\left({ }^{54} \mathrm{Fe}\right)+1 / 29 \mathrm{X}\left({ }^{58} \mathrm{Ni}\right)$ and $\mathrm{X}\left({ }^{58} \mathrm{Ni}\right)=\mathrm{f} \mathrm{X}\left({ }^{54} \mathrm{Fe}\right)$ with $\mathrm{f}=$ 0.35 (Hartmann, Woosley \& El Eid 1985). If X(He) is larger than 20 percent, however, the so-called $\alpha$-rich freeze out is assumed to produce mostly ${ }^{58} \mathrm{Ni}$ so that $\mathrm{f}=10$. For $\eta$ less than $1 / 14$, but greater than $1 / 27, \mathrm{X}\left({ }^{56} \mathrm{Ni}\right)=0$ and the sum of the ${ }^{54} \mathrm{Fe},{ }^{56} \mathrm{Fe}$, and ${ }^{58} \mathrm{Ni}$ mass fractions is $1-\mathrm{X}(\mathrm{He})$. Neutron conservation additionally gives $\eta=1 / 27 \mathrm{X}\left({ }^{54} \mathrm{Fe}\right)+1 / 29$ $\mathrm{X}\left({ }^{58} \mathrm{Ni}\right)+1 / 14 \mathrm{X}\left({ }^{56} \mathrm{Fe}\right)$, and the condition $\mathrm{X}\left({ }^{58} \mathrm{Ni}\right)=\mathrm{f} \mathrm{X}\left({ }^{54} \mathrm{Fe}\right)$, as before, can be solved to give the 3 isotopic abundances separately. For $\eta$ greater than $1 / 14$, which was only true for a small fraction of the matter where burning happened at very high density, ${ }^{56} \mathrm{Fe}$ was assumed to be the only isotope for iron group elements. 


\section{Results}

Figure 3 shows the propagation of the flame front at different star times for our standard run, model A100. The flame is wrinkled and distorted by the Rayleigh-Taylor instability, with buoyant hot ash bubbles floating toward the surface and spreading while cold, denser matter moves outwards more slowly and is overtaken. Viewed in Lagrangian coordinates, the unburned matter appears to migrate inwards. At very early times, one sees finger-like structures resulting from the initial perturbations, but these quickly mushroom and become non-linear. New plumes form and the structure becomes complex.

During the early growth of the reactive RT instability, burning effectively smooths out perturbations below the fire polishing length (Equation (3)). As the RT modes begin to merge, interact, and break down, they become wrinkled on ever smaller scales (Zingale et al. 2005, e.g.). Indeed, the flame experiences the effects of turbulence on scales down to the Gibson scale - the scale at which the laminar speed equals the turbulent speed. Furthermore, the turbulent scaling study of Ciaraldi-Schoolmann et al. (2009) shows that the nature of the turbulence shifts from being dominated by RT scaling (Equation (3) ) to Kolmogorov scaling for isotropic eddies on scales smaller than $\sim 15 \mathrm{~km}$, or about the initial $\lambda_{\min }$. It should be noted that with our choice of grid resolution in the present studies, we are not resolving the turbulent flame on scales smaller than $\lambda_{\min }$.

Several effects lead to the evolution of $\lambda_{\min }$. As the flame moves out, the greater interior mass enclosed tends to increase the gravitational acceleration, but at the same time the star expands, causing it to decrease. The density contrast, $\Delta \rho / \rho$ also gets larger at lower density. The combined effect is to decrease $g_{\text {eff }}$ slowly, though its value remains roughly constant at about $10^{9} \mathrm{~cm} \mathrm{~s}^{-2}$ throughout much of the burning. Since the flame speed is also a constant, $100 \mathrm{~km} \mathrm{~s}^{-1}$ in this case, $\lambda_{\min }$ does not vary greatly from about $10 \mathrm{~km}$. While there are always at least several zones within $\lambda_{\text {min }}$, this does not necessarily mean that structures of this scale are well resolved. Since the thickened flame itself is typically a few to five zones wide and any structure must be bounded on all sides where there is fuel by flames, $\lambda_{\min }$ is only marginally resolved. The smallest structures in Figure 6 are roughly $40 \mathrm{~km}$ across. The length scale $\lambda_{\min }$ is more fully resolved for the model A200 run, but less resolved for model A50. Ideally, greater resolution should be employed, but it would be prohibitively expensive to run with even twice the current resolution since run time scales as the fourth power of the resolution, three for the three dimensions plus one for the shorter Courant time step. Fortunately, the most interesting burning happens on the finest grid scale, which is 1 to $2 \mathrm{~km}$ during the nucleosynthesis epoch. By $1.2 \mathrm{~s}$ when the finest grid becomes $4 \mathrm{~km}$,

$g \sim 8 \times 10^{8} \mathrm{~cm} \mathrm{~s}^{-2}$ and $\Delta \rho / \rho \sim 0.3$, so $\lambda_{\min }$ has already increased to $50 \mathrm{~km}$. Visually, one also sees more structure in the model A50 run (Figure 6), suggesting that the structure is 
sensitive to $\mathrm{V}$ and we are actually resolving $\lambda_{\min }$.

The flame first breaks out of the surface in the model A100 run $1.21 \mathrm{~s}$ after ignition, by which time the star has already expanded by a factor of about 2.5 and the central density has declined to $5 \times 10^{7} \mathrm{~g} \mathrm{~cm}^{-3}$. This decrease in density significantly affects the products of the burning and allows the production of significant intermediate mass elements. The largest speed up to this point is $\sim 10,000 \mathrm{~km} \mathrm{~s}^{-1}$ in the outermost layers of the star, but the flow is still subsonic in most of the star. Nuclear burning is over at this point and, as the internal energy converts into kinetic energy, the maximum speed rises to $25,000 \mathrm{~km} \mathrm{~s}^{-1}$ at $2.76 \mathrm{~s}$.

Figure 4 shows how kinetic energy, binding energy and internal energy evolve with time. At the beginning of the explosion, the net binding energy, i.e., the gravitational binding energy minus the internal energy, is about $5 \times 10^{50}$ ergs. The final kinetic energy at infinity is about $6 \times 10^{50} \mathrm{ergs}$, so the total energy released thermonuclear burning energy is $1.1 \times 10^{51}$ ergs. The supernova ejecta is mainly composed of $0.63 \mathrm{M}_{\odot}$ iron group elements and $0.16 \mathrm{M}_{\odot}$ IME. The mass of ${ }^{56} \mathrm{Ni}$ and the final kinetic energy suggests a relatively weak event in SN Ia.

A sequence of plots in Figure 5 shows the evolution of the angle-averaged supernova composition. At early time, iron group elements are the major products, but after the flame spreads to the low density region (less than $5 \times 10^{7} \mathrm{~g} \mathrm{~cm}^{-3}$ ) at $1 \mathrm{~s}$, more IME are made. As the iron-group ashes continue to move out at higher speeds than the unburned fuel, boosted along by the recombination of helium, there is a gradual accumulation of carbon and oxygen near the center of the explosion. At the end, about $20 \%$ unburned carbon and oxygen are left in the central region.

The calculation was repeated using two different flame speed parameters, models A50 and A200, and followed until $1.8 \mathrm{~s}$ when the nuclear burning was over (but not into the final coasting phase). Comparisons of the flame front morphology using the different flame speeds are shown in Figure 6. Slower flame speeds result in increased structure as noted, but as Figure 7 shows, the the total amount of burned material is roughly the same for flame speeds of $100 \mathrm{~km} \mathrm{~s}^{-1}$ and $200 \mathrm{~km} \mathrm{~s}^{-1}$. The slower burning speed is compensated for by a larger surface area. For a flame speed of $50 \mathrm{~km} \mathrm{~s}^{-1}$, the calculation produces $0.73 \mathrm{M}_{\odot}$ iron, $0.17 \mathrm{M}_{\odot}$ IME, and released $1.3 \times 10^{51} \mathrm{ergs}$. This is significantly more burning than either of the other two series A runs but still within $10 \%$ of their average. We do not know if this limited variation is due to the small number of runs or reflects real physical dependence on the parameter. There is a tendency, however, for more burning to occur in an explosion that starts slower because it remains denser longer. 
The B series simulations used a different random number generator seed than the A series simulations; the random number generator applied spherical perturbations of random size and amplitude to the initial flame surface. Figure 8 compares the $X\left({ }^{12} \mathrm{C}\right)=0.49$ isosurface of the constant flame speed model B100 (left) to that of the turbulent flame speed model BT (right) at $0.3 \mathrm{~s}$ (top), $0.5 \mathrm{~s}$ (middle), and 0.7s (bottom). Morphologically, models B100 and BT are nearly identical. A comparison of the left column of Figure 8 (B100) with the center column of Figure 6] (A100) at the same time elucidates the difference in initial model conditions between the two series. The A series perturbations are all nearly of the same magnitude, whereas the B series initially has a few slightly dominant perturbations that grow more rapidly and develop into large-scale RT plumes; this feature is similar to published $2 \mathrm{~d}$ studies of central ignition with perturbed spheres (Jackson et al. 2010; Krueger et al. 2012, for example). The extraordinarily large appearance of the plume in the lower edge of the plots is a result of that plume's coming towards the viewer. Neglecting the dominant plumes, at any given time the size of the ash bubble is about the same in all runs.

Figure 9 shows the total mass of iron group elements (red) and IME (blue) produced for the B series of models. The dotted lines are for model B100, and the dashed lines are model BT. The two thin, solid lines show the percent difference between the two models where positive (negative) values indicate model B100 producing more (less) material than model BT. These two different flame models agree remarkably well. Initially, the constant flame speed for model B100 is greater than the laminar flame speed, which is the speed at which model BT burns in the absence of turbulence. This difference causes model B100 to burn more material more rapidly than model BT. By about $0.2 \mathrm{~s}$, some zones within the flame experience turbulent velocities that are greater than the local laminar flame speed and/or the constant $100 \mathrm{~km} \mathrm{~s}^{-1}$ flame speed of model B100; the flame in model BT begins to increase in speed, which burns more material that then decreases the difference between the two flame prescriptions. As the flame grew in size, the number of fine-resolution zones increased. After $\sim 0.31 \mathrm{~s}$ of evolution, there were over one billion zones in the finest level and we de-refined the simulation for computational efficiency. This increase by a factor of two in zone size (in each direction) caused a corresponding increase in the turbulent intensity on the grid scale by a factor of $2^{1 / 3}$ according to the Kolmogorov scaling we assumed in our turbulent flame speed model. This is evident in Figure 9 as a small but sudden increase in the percent difference between elemental yields with the constant flame speed model and the turbulent flame speed model. Another de-refinement occurred around $0.61 \mathrm{~s}$, but the change in percent difference between the models is lost in the noise.

Around $0.4 \mathrm{~s}$, the turbulent flame in model BT has "caught up" to the constant flame speed model B100 in terms of total mass produced in each elemental group. The probability density functions for the turbulent speed at various times in Figure 10 show that indeed the 
most likely turbulent flame speed used around this time in model BT is $\sim 100 \mathrm{~km} \mathrm{~s}^{-1}$ as is used in model B100. The distribution does have an extended tail, which shows some subset of the zones within the flame experience turbulent intensities greater than the constant flame speed model B100. This causes the turbulent flame speed model BT to overproduce iron and IME relative to model B100 (negative percent differences in Figure9). It is around this point in the evolution that simple interpretation of the differences in iron and IME yields between the two flame speed prescriptions becomes difficult. There is a constant battle between 1) the small differences in flame structure being amplified by the (dominant) acceleration due to buoyancy, 2) the dominant plumes reaching a region where the density gradient becomes steep, and 3) the shear induced turbulence that tends to redistribute the turbulent flame speed to ever higher velocities. Even though the total mass of iron and IME is increasing in both models, the differences between model B100 and BT fluctuate between positive and negative due to the interplay of the above mentioned forces.

Throughout the B series runs, the choice of flame speed model (constant vs. turbulent) made less than a $7 \%$ difference in the iron and IME group total yields. Comparing, however, slightly different initial conditions but the same (constant) flame speed model-models A100 (Figure 7) and B100 (Figure 10) - at, say, 0.6 s shows nearly a 50\% difference in total iron group yield. This seems to suggest that the simulation results are much more sensitive to the initial conditions of the hot ash than they are to the flame speed prescription, at least in the case of a constant flame speed model and a model where the flame speed depends simply on the turbulent intensity on the grid within the flame.

\section{Discussion and Conclusions}

The elemental yields of our models and the nuclear energy released by each are given in Table 6. It is important to note that the B series models were not run to completion so their tabulated elemental yields should not be compared with the other models in the table, but only with each other to assess the role of a simplified turbulent flame model. Results from the A series studies with $\mathrm{V}=100$ and $200 \mathrm{~km} \mathrm{~s}^{-1}$ resemble each other closely and are also similar to those of Röpke et al. (2006). They do show, however, significantly less production of intermediate mass elements than Röpke et al. (2007a). We do not know the specific cause of the large production of intermediate mass elements in the Röpke study, but note that they are significantly larger than in any other study. Perhaps this reflects the initial conditions and igniting a very large number of ignition points or an unusually high flame speed from the turbulent subgrid model at low densities (see below). García-Senz \& Bravo (2005) found around $0.45 \mathrm{M}_{\odot}^{56} \mathrm{Ni}$ was produced in the explosion with $\sim 15$ initial igniting 
bubbles near the center, which is similar to our series A results, but IME production in their simulation was much smaller. Gamezo et al. (2005) found only $0.47 \mathrm{M}_{\odot}$ iron group elements were produced during the deflagration assuming an initial ignition region with a radius of only $30 \mathrm{~km}$.

The actual mass of ${ }^{56} \mathrm{Ni}$ implied by observations of SN Ia is highly variable ranging from 0.1 to $0.9 \mathrm{M}_{\odot}$ (Mazzali et al. 2007), but a typical value is about $0.6 \mathrm{M}_{\odot}$ (Branch \& Khokhlov 1995). A lower limit to the total burned mass derived from observations of the velocity of silicon, applying the density-velocity structure in W7 model, is around $1.05 \mathrm{M}_{\odot}$ (Mazzali et al. 2007). Our series A calculations found that approximately $0.9-1.0 \mathrm{M}_{\odot}$ of the $1.38 \mathrm{M}_{\odot}$ white dwarf was burned to heavier elements regardless of the assumed flame speed, not too much smaller than the optimal value. This burning certainly released enough energy to unbind the star, but is well short of what is needed to account for the brighter SN Ia.

Perhaps this is not too surprising. If typical SN Ia ignite off center and later detonate, then one expects a correlation between the displacement of the ignition and the brightness of the supernova. Explosions igniting off-center burn less before detonating and are thus denser when the detonation occurs. They thus make more ${ }^{56} \mathrm{Ni}$ and are brighter. Central ignition is a limiting case where the burning in the deflagration stage is maximal. A subsequent detonation makes less ${ }^{56} \mathrm{Ni}$ and the total synthesis may be less, hence a fainter supernova. The puzzle then is not why deflagrations are faint, but the origin of some SN Ia that are fainter still.

The main difficulty faced by pure deflagrations though is not their brightness, but their spectrum. They have too much low velocity unburned fuel near their middles (Marion et al. 2003). More than $70 \%$ of the central ejecta in our standard model A100 was carbon and oxygen. Even though the central regions initially burned to completion, unburned fuel is later found near the center of the star because the ashes have floated above the fuel, leaving it behind as they move outward with greater speed than their cold surroundings (see Figure 11). Iron group elements and IME in the ejecta continue mixing radially even after burning has gone out; the layered structure of different chemical products present at the end of burning but before much expansion is hardly seen. This is contrary to the observed spectral lines of carbon and silicon in the supernova ejecta, which indicates velocities mostly beyond 10,000 $\mathrm{km} \mathrm{s}^{-1}$ (Filippenko 1997; Branch et al. 2003; Benetti et al. 2005; Parrent et al. 2011). Previous numerical studies have demonstrated the same shortcomings out of the centrally-ignited pure deflagration model. For example, Gamezo et al. (2003) assumed a centrally burned region of radius $30 \mathrm{~km}$, and carried out the following deflagration simulation with grid-length and effective gravity-dependent flame speeds in the thick-flame model. $80 \%$ to $90 \%$ percent of the low velocity materials near the center are composed of carbon and oxygen at $1.9 \mathrm{~s}$ in 
their study. Other numerical works initialized a number of hot bubbles around the center instead of the whole central region, including 2D and 3D studies using a different flame tracking method and subgrid model (Reinecke, Hillebrandt \& Niemeyer 2002; Röpke et al. 2006), and 3D calculations using a smoothed particle hydrodynamics (SPH) code (García-Senz \& Bravo 2005). Central carbon concentrations were found in all these studies. However, parameter studies have shown that larger numbers (more than 150) of initially Gaussian distributed hot bubbles with a maximum ignition radius of $180 \mathrm{~km}$ leave much less unburned fuel in central regions, resulting a spectrum better consistent with the observations (Röpke et al. 2006, 2007a). This specific ignition pattern is unlikely to happen at least in mildly turbulent convective core of the white dwarf though (Kuhlen, Woosley \& Glatzmaier 2006; Zingale et al. 2009, 2011).

This weakness of the pure deflagration model might be alleviated by a later transition from deflagration to detonation (DDT) in the center or at a certain radius (Gamezo et al. 2005). The supersonic detonation wave is expected to burn off the inhomogeneous unburned materials, release enough energy to match classic SN Ia, and give stratified shells of product elements. The synthetic light curves and spectra of the DDT explosion agreed well with the observations of a normal SN Ia (Kasen et al. 2009). It has been suspected that a DDT may take place when the flame enters the distributed burning regime at the density of $1 \sim$ $3 \times 10^{7} \mathrm{~g} \mathrm{~cm}^{-3}$, where turbulence starts to deform the internal flame structure and mix the hot ashes and fuel (Niemeyer \& Woosley 1997). The existence of strong turbulence, with velocity fluctuations larger than one fifth of local sound speed, is crucial for a spontaneous detonation (Woosley et al. 2009), and Röpke (2007) suggested the possibility of these high turbulent velocities at the corresponding density range from their deflagration simulations. We plan to study the outcome of a later detonation following the current deflagration calculations with 2 free parameters, the location and time of the detonation wave initiation.

We thank Andy Aspden and Mike Zingale for helpful discussions regarding the turbulent flame physics and the set up of problems in hydrostatic equilibrium using the CASTRO code. The work at LBNL was supported by the SciDAC Program of the DOE Office of High Energy Physics and by the Applied Mathematics Program of the DOE Office of Advance Scientific Computing Research under U.S. Department of Energy under contract No. DEAC02-05CH11231. The work at UCSC was supported by the DOE SciDAC program, under grant No. DE-FC02-06ER41438 and by the NASA Theory Program (NNX09AK36G). Computer time for the calculations in this paper was provided through a DOE INCITE award at the Oak Ridge Leadership Computational Facility (OLCF) at Oak Ridge National Laboratory, which is supported by the Office of Science of the U.S. Department of Energy under Contract No. DE-AC05-00OR22725. This research used resources of the National Energy 
Research Scientific Computing Center, which is supported by the Office of Science of the U.S. Department of Energy under Contract No. DE-AC02-05CH11231. Early developmental work necessary to this study was carried out on the Pleiades supercluster at UCSC. Pleiades was purchased using a grant from the MRI Program of the NSF (AST-0521566). Visualizations were performed using the VisIt package. We thank Gunther Weber and Hank Childs for their assistance with VisIt. 
Table 1: Final composition - deflagration ashes

\begin{tabular}{cccccccc}
\hline \hline $\begin{array}{c}\text { Log density } \\
\left(\mathrm{g} \mathrm{cm}^{-3}\right)\end{array}$ & $\mathrm{He}$ & $\mathrm{C}$ & $\mathrm{O}$ & $\mathrm{Ne}$ & $\mathrm{Mg}$ & $\mathrm{Si}$ & $\mathrm{Fe}$ \\
\hline 6.40 & - & 0.056 & 0.341 & 0.411 & 0.177 & 0.015 & - \\
6.50 & - & 0.050 & 0.348 & 0.395 & 0.189 & 0.019 & - \\
6.60 & - & 0.037 & 0.358 & 0.373 & 0.208 & 0.024 & - \\
6.70 & - & 0.014 & 0.399 & 0.285 & 0.255 & 0.046 & - \\
6.80 & - & 0.002 & 0.549 & 0.012 & 0.244 & 0.194 & - \\
6.90 & - & - & 0.560 & - & 0.213 & 0.227 & - \\
7.00 & - & - & 0.561 & - & 0.194 & 0.245 & - \\
7.10 & - & - & 0.548 & - & 0.167 & 0.285 & - \\
7.20 & - & - & 0.382 & - & 0.031 & 0.587 & - \\
7.30 & - & - & - & - & - & 0.992 & 0.007 \\
7.40 & 0.001 & - & - & - & - & 0.971 & 0.028 \\
7.50 & 0.003 & - & - & - & - & 0.825 & 0.171 \\
7.60 & 0.013 & - & - & - & - & 0.370 & 0.617 \\
7.70 & 0.030 & - & - & - & - & 0.079 & 0.891 \\
7.80 & 0.048 & - & - & - & - & 0.033 & 0.918 \\
7.90 & 0.064 & - & - & - & - & 0.037 & 0.899 \\
8.00 & 0.081 & - & - & - & - & 0.040 & 0.879 \\
8.10 & 0.097 & - & - & - & - & 0.044 & 0.860 \\
8.20 & 0.112 & - & - & - & - & 0.047 & 0.842 \\
8.30 & 0.125 & - & - & - & - & 0.050 & 0.825 \\
8.40 & 0.137 & - & - & - & - & 0.054 & 0.809 \\
8.50 & 0.148 & - & - & - & - & 0.057 & 0.795 \\
8.60 & 0.157 & - & - & - & - & 0.061 & 0.781 \\
8.70 & 0.166 & - & - & - & - & 0.066 & 0.769 \\
8.80 & 0.173 & - & - & - & - & 0.070 & 0.757 \\
8.90 & 0.179 & - & - & - & - & 0.075 & 0.746 \\
9.00 & 0.185 & - & - & - & - & 0.080 & 0.735 \\
9.10 & 0.190 & - & - & - & - & 0.085 & 0.725 \\
9.20 & 0.194 & - & - & - & - & 0.091 & 0.715 \\
9.30 & 0.198 & - & - & - & 0.001 & 0.097 & 0.705 \\
9.40 & 0.201 & - & - & - & 0.001 & 0.103 & 0.695 \\
9.50 & 0.203 & - & - & - & 0.001 & 0.110 & 0.685 \\
9.60 & 0.205 & - & - & - & 0.001 & 0.118 & 0.675 \\
9.70 & 0.207 & - & 0.001 & - & 0.001 & 0.126 & 0.665 \\
9.80 & 0.208 & - & 0.001 & - & 0.001 & 0.135 & 0.655 \\
9.90 & 0.208 & 0.001 & 0.001 & - & 0.002 & 0.144 & 0.644 \\
10.0 & 0.207 & 0.001 & 0.001 & - & 0.002 & 0.156 & 0.639 \\
\hline & & & & & & &
\end{tabular}


Table 2: Bulk properties - deflagration ashes

\begin{tabular}{|c|c|c|c|c|c|}
\hline $\begin{array}{c}\log \rho \\
\left(\mathrm{g} \mathrm{cm}^{-3}\right)\end{array}$ & $\begin{array}{c}T_{9 f} \\
\left(10^{9} \mathrm{~K}\right)\end{array}$ & $\begin{array}{c}\rho_{7 f} \\
\left(10^{7} \mathrm{~g} \mathrm{~cm}^{-3}\right)\end{array}$ & $\begin{array}{c}\mathrm{d} \rho_{7} / \mathrm{d} \mathrm{X}_{12} \\
\left(10^{7} \mathrm{~g} \mathrm{~cm}^{-3}\right)\end{array}$ & $\begin{array}{c}\mathrm{BE} / \mathrm{A} \\
(\mathrm{MeV} / \text { nucleon })\end{array}$ & $\overline{\bar{A}}$ \\
\hline 6.40 & 1.790 & 0.122 & 0.258 & 8.040 & 18.340 \\
\hline 6.50 & 1.903 & 0.158 & 0.316 & 8.046 & 18.430 \\
\hline 6.60 & 2.036 & 0.203 & 0.390 & 8.057 & 18.630 \\
\hline 6.70 & 2.216 & 0.255 & 0.493 & 8.082 & 18.980 \\
\hline 6.80 & 2.471 & 0.305 & 0.651 & 8.137 & 19.190 \\
\hline 6.90 & 2.631 & 0.399 & 0.792 & 8.144 & 19.240 \\
\hline 7.00 & 2.791 & 0.522 & 0.955 & 8.147 & 19.280 \\
\hline 7.10 & 2.978 & 0.677 & 1.163 & 8.158 & 19.460 \\
\hline 7.20 & 3.382 & 0.794 & 1.582 & 8.262 & 21.680 \\
\hline 7.30 & 3.899 & 0.878 & 2.235 & 8.464 & 29.270 \\
\hline 7.40 & 4.154 & 1.158 & 2.708 & 8.467 & 29.440 \\
\hline 7.50 & 4.444 & 1.507 & 3.310 & 8.488 & 30.480 \\
\hline 7.60 & 4.781 & 1.932 & 4.099 & 8.527 & 31.730 \\
\hline 7.70 & 5.071 & 2.533 & 4.959 & 8.518 & 28.210 \\
\hline 7.80 & 5.311 & 3.375 & 5.869 & 8.479 & 23.970 \\
\hline 7.90 & 5.542 & 4.489 & 6.909 & 8.442 & 21.130 \\
\hline 8.00 & 5.761 & 5.948 & 8.104 & 8.406 & 19.000 \\
\hline 8.10 & 5.969 & 7.848 & 9.482 & 8.373 & 17.410 \\
\hline 8.20 & 6.169 & 10.31 & 11.08 & 8.343 & 16.190 \\
\hline 8.30 & 6.362 & 13.49 & 12.93 & 8.316 & 15.240 \\
\hline 8.40 & 6.550 & 17.58 & 15.08 & 8.293 & 14.500 \\
\hline 8.50 & 6.736 & 22.83 & 17.60 & 8.272 & 13.910 \\
\hline 8.60 & 6.921 & 29.54 & 20.54 & 8.254 & 13.440 \\
\hline 8.70 & 7.105 & 38.13 & 23.98 & 8.238 & 13.050 \\
\hline 8.80 & 7.291 & 49.09 & 28.02 & 8.224 & 12.720 \\
\hline 8.90 & 7.479 & 63.05 & 32.76 & 8.211 & 12.450 \\
\hline 9.00 & 7.670 & 80.84 & 38.33 & 8.200 & 12.220 \\
\hline 9.10 & 7.866 & 103.50 & 44.87 & 8.190 & 12.020 \\
\hline 9.20 & 8.065 & 132.20 & 52.57 & 8.181 & 11.860 \\
\hline 9.30 & 8.270 & 168.70 & 61.63 & 8.173 & 11.720 \\
\hline 9.40 & 8.480 & 215.00 & 72.30 & 8.166 & 11.600 \\
\hline 9.50 & 8.697 & 273.80 & 84.88 & 8.160 & 11.500 \\
\hline 9.60 & 8.922 & 348.30 & 99.71 & 8.154 & 11.420 \\
\hline 9.70 & 9.153 & 442.60 & 117.20 & 8.149 & 11.360 \\
\hline 9.80 & 9.393 & 562.00 & 137.90 & 8.145 & 11.310 \\
\hline 9.90 & 9.641 & 713.20 & 162.30 & 8.141 & 11.270 \\
\hline 10.0 & 10.090 & 902.10 & 195.80 & 8.194 & 11.260 \\
\hline
\end{tabular}


Table 3: Comparison with published flame models

\begin{tabular}{cccccc}
\hline \hline $\begin{array}{c}\text { Log density } \\
\left(\mathrm{g} \mathrm{cm}^{-3}\right)\end{array}$ & $\begin{array}{c}\mathrm{T}_{\text {ash }} \\
\left(10^{9} \mathrm{~K}\right)\end{array}$ & $\begin{array}{c}\text { Pub. } \mathrm{T}_{a s h} \\
\left(10^{9} \mathrm{~K}\right)\end{array}$ & $\Delta \rho / \rho$ & Pub. $\Delta \rho / \rho$ & Ref. \\
\hline 6.78 & 2.42 & 2.45 & 0.514 & 0.527 & Woo07 \\
7.0 & 2.79 & 2.79 & 0.478 & 0.477 & Woo07 \\
$7.398^{*}$ & 4.11 & 4.1 & 0.529 & - & Woo11 \\
$7.477^{*}$ & 4.33 & 4.3 & 0.515 & - & Woo11 \\
8.0 & 5.76 & - & 0.405 & 0.426 & Tim92 \\
9.0 & 7.67 & - & 0.192 & 0.192 & Tim92 \\
10.0 & 10.1 & - & 0.098 & 0.094 & Tim92 \\
\hline
\end{tabular}


Table 4: Sample characteristics of nuclear statistical equilibrium

\begin{tabular}{ccccccccc}
\hline \hline Log T & Log density & $Y_{e}$ & $\mathrm{X}(\mathrm{He})$ & $\mathrm{X}(\mathrm{Si}-\mathrm{Ca})$ & $\mathrm{X}$ (Fe group) & $\bar{A}$ & $\mathrm{BE} / \mathrm{A}$ & $\mathrm{d} Y_{e} / \mathrm{dt}$ \\
\hline 9.80 & 9.30 & 0.500 & $1.601(-2)$ & $5.174(-2)$ & 0.9322 & 35.29 & 8.55635 & $2.617(-1)$ \\
9.80 & 9.30 & 0.495 & $1.205(-2)$ & $4.097(-2)$ & 0.9469 & 40.79 & 8.60340 & $1.920(-1)$ \\
9.80 & 9.30 & 0.490 & $9.798(-3)$ & $2.832(-2)$ & 0.9618 & 44.58 & 8.63952 & $1.313(-1)$ \\
9.80 & 9.30 & 0.485 & $8.243(-3)$ & $1.645(-2)$ & 0.9752 & 47.26 & 8.66989 & $8.148(-2)$ \\
9.80 & 9.30 & 0.480 & $7.000(-3)$ & $7.951(-3)$ & 0.9850 & 49.21 & 8.69495 & $4.668(-2)$ \\
9.80 & 9.30 & 0.475 & $5.975(-3)$ & $3.575(-3)$ & 0.9904 & 50.57 & 8.71502 & $2.645(-2)$ \\
9.80 & 9.30 & 0.470 & $5.042(-3)$ & $1.601(-3)$ & 0.9933 & 51.64 & 8.73201 & $1.449(-2)$ \\
9.80 & 9.30 & 0.465 & $4.072(-3)$ & $7.147(-4)$ & 0.9952 & 52.58 & 8.74541 & $7.197(-3)$ \\
9.80 & 9.30 & 0.460 & $3.159(-3)$ & $3.524(-4)$ & 0.9964 & 53.37 & 8.75220 & $3.470(-3)$ \\
9.80 & 9.30 & 0.455 & $2.439(-3)$ & $1.989(-4)$ & 0.9973 & 53.96 & 8.75367 & $1.745(-3)$ \\
9.80 & 9.30 & 0.450 & $1.841(-3)$ & $1.115(-4)$ & 0.9980 & 54.40 & 8.75201 & $8.264(-4)$ \\
9.80 & 9.30 & 0.445 & $1.339(-3)$ & $5.506(-5)$ & 0.9986 & 54.74 & 8.74627 & $3.423(-4)$ \\
9.80 & 9.30 & 0.440 & $1.001(-3)$ & $2.451(-5)$ & 0.9989 & 55.16 & 8.73595 & $1.219(-4)$ \\
9.80 & 9.30 & 0.435 & $8.112(-4)$ & $1.029(-5)$ & 0.9991 & 55.70 & 8.72337 & $1.182(-5)$ \\
9.80 & 9.30 & 0.430 & $7.294(-4)$ & $3.667(-6)$ & 0.9992 & 56.02 & 8.70984 & $-5.946(-5)$ \\
9.80 & 9.30 & 0.425 & $8.355(-4)$ & $8.179(-7)$ & 0.9991 & 55.10 & 8.69408 & $-1.119(-4)$ \\
9.80 & 9.30 & 0.420 & $1.933(-3)$ & $6.037(-8)$ & 0.9980 & 49.51 & 8.66726 & $-1.310(-4)$ \\
\hline
\end{tabular}


Table 5: Flame adjustment parameters, $f_{w}, f_{v}$, at different densities

\begin{tabular}{c|cc}
$\log (\rho)$ & $f_{w}$ & $f_{v}$ \\
\hline 6.90 & 2.40 & 2.90 \\
7.00 & 2.00 & 2.20 \\
7.15 & 1.80 & 1.95 \\
7.30 & 1.40 & 1.55 \\
7.50 & 1.00 & 1.20 \\
7.70 & 1.00 & 1.05 \\
7.85 & 1.20 & 1.10 \\
8.00 & 1.20 & 1.15 \\
8.30 & 1.60 & 1.30 \\
8.50 & 1.80 & 1.40 \\
8.70 & 2.00 & 1.60 \\
8.85 & 2.20 & 1.70 \\
9.00 & 2.40 & 1.75 \\
9.20 & 2.80 & 2.00 \\
9.30 & 3.00 & 2.20 \\
9.40 & 3.40 & 2.30 \\
9.45 & 3.40 & 2.30
\end{tabular}


Table 6: Mass of chemical products and the total released energy

\begin{tabular}{ccccccccccccc}
\hline \hline Model & $\begin{array}{c}\mathrm{M}(\mathrm{C}) \\
\left(\mathrm{M}_{\odot}\right)\end{array}$ & $\begin{array}{c}\mathrm{M}(\mathrm{O}) \\
\left(\mathrm{M}_{\odot}\right)\end{array}$ & $\begin{array}{c}\mathrm{M}(\mathrm{He}) \\
\left(\mathrm{M}_{\odot}\right)\end{array}$ & $\begin{array}{c}\mathrm{M}(\mathrm{Ne}) \\
\left(\mathrm{M}_{\odot}\right)\end{array}$ & $\begin{array}{c}\mathrm{M}(\mathrm{Mg}) \\
\left(\mathrm{M}_{\odot}\right)\end{array}$ & $\begin{array}{c}\mathrm{M}(\mathrm{IME}) \\
\left(\mathrm{M}_{\odot}\right)\end{array}$ & $\begin{array}{c}\mathrm{M}(" \mathrm{Fe}) \\
\left(\mathrm{M}_{\odot}\right)\end{array}$ & $\begin{array}{c}\mathrm{M}\left({ }^{54} \mathrm{Fe}\right) \\
\left(\mathrm{M}_{\odot}\right)\end{array}$ & $\begin{array}{c}\mathrm{M}\left({ }^{56} \mathrm{Fe}\right) \\
\left(\mathrm{M}_{\odot}\right)\end{array}$ & $\begin{array}{c}\mathrm{M}\left({ }^{56} \mathrm{Ni}\right) \\
\left(\mathrm{M}_{\odot}\right)\end{array}$ & $\begin{array}{c}\mathrm{M}\left({ }^{58} \mathrm{Ni}\right) \\
\left(\mathrm{M}_{\odot}\right)\end{array}$ & $\begin{array}{c}\mathrm{E}_{n u c} \\
\left(10^{51} \mathrm{ergs}\right)\end{array}$ \\
\hline $\mathrm{A} 50$ & 0.17 & 0.24 & 0.04 & 0.006 & 0.03 & 0.17 & 0.73 & 0.12 & 0.0012 & 0.56 & 0.04 & 1.26 \\
$\mathrm{~A} 100$ & 0.22 & 0.30 & 0.05 & 0.005 & 0.03 & 0.16 & 0.63 & 0.11 & 0.0005 & 0.48 & 0.04 & 1.15 \\
$\mathrm{~A} 200$ & 0.21 & 0.29 & 0.03 & 0.006 & 0.03 & 0.17 & 0.65 & 0.11 & 0.0015 & 0.50 & 0.04 & 1.16 \\
$\mathrm{~B} 100^{\mathrm{a}}$ & 0.48 & 0.48 & 0.04 & $4.0(-7)$ & $5.5(-5)$ & 0.02 & 0.37 & - & - & - & - & - \\
BT $^{\mathrm{a}}$ & 0.47 & 0.47 & 0.04 & $1.5(-6)$ & $6.2(-5)$ & 0.02 & 0.38 & - & - & - & - & - \\
b150_3d $^{b}$ & 0.28 & 0.28 & - & - & - & 0.17 & 0.67 & - & - & - & - & 1.18 \\
b250_3d $^{\mathrm{b}}$ & 0.31 & 0.31 & - & - & - & 0.16 & 0.61 & - & - & - & - & 1.09 \\
SNOB $^{\mathrm{c}}$ & 0.18 & 0.18 & - & - & - & 0.43 & 0.61 & - & - & 0.33 & - & 1.31 \\
\hline
\end{tabular}

${ }^{a}$ Not run to burning completion — only out to $0.74 \mathrm{~s}$; should only be used for comparison between B series simulations

[Röpke et al. (2006)

Röpke et al. (2007a) 


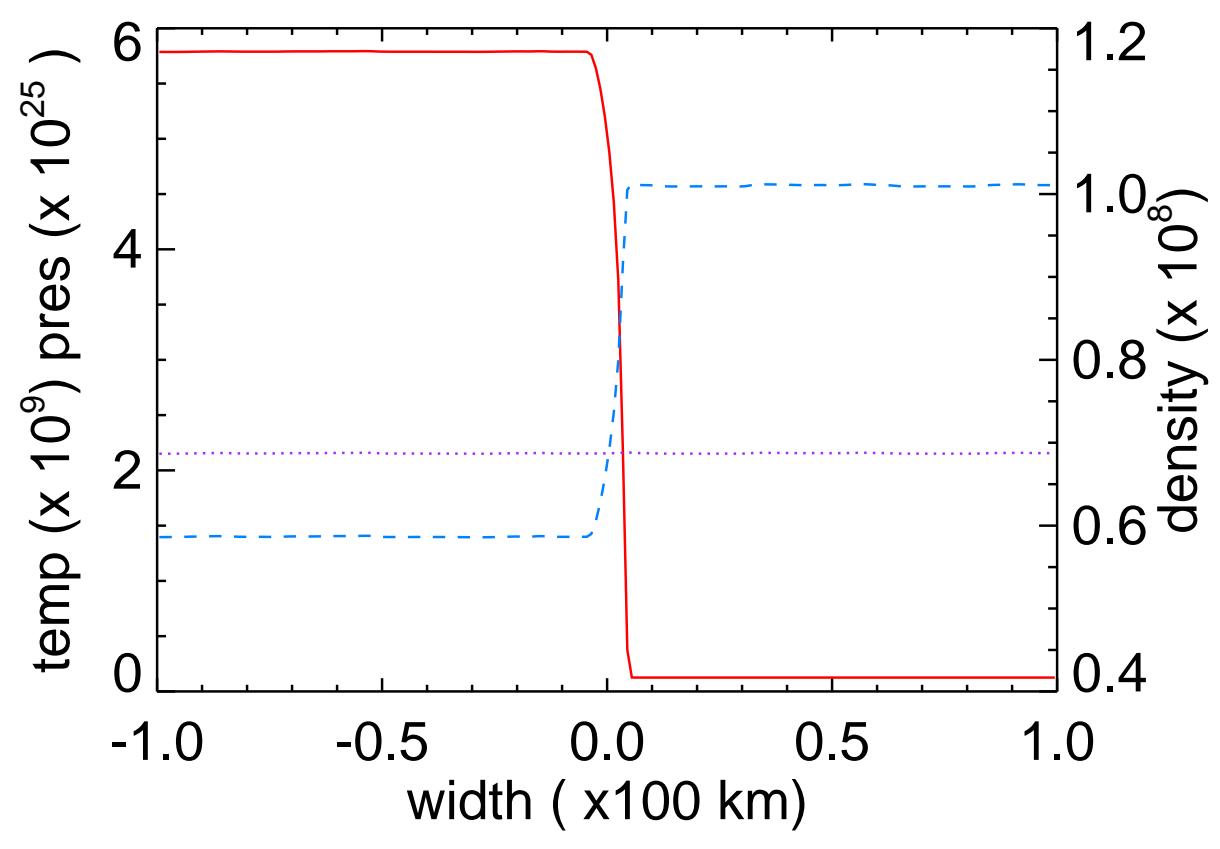

Fig. 1.- Density (dashed), temperature (solid) and pressure (dotted) profiles across the 1D laminar flame surface. 


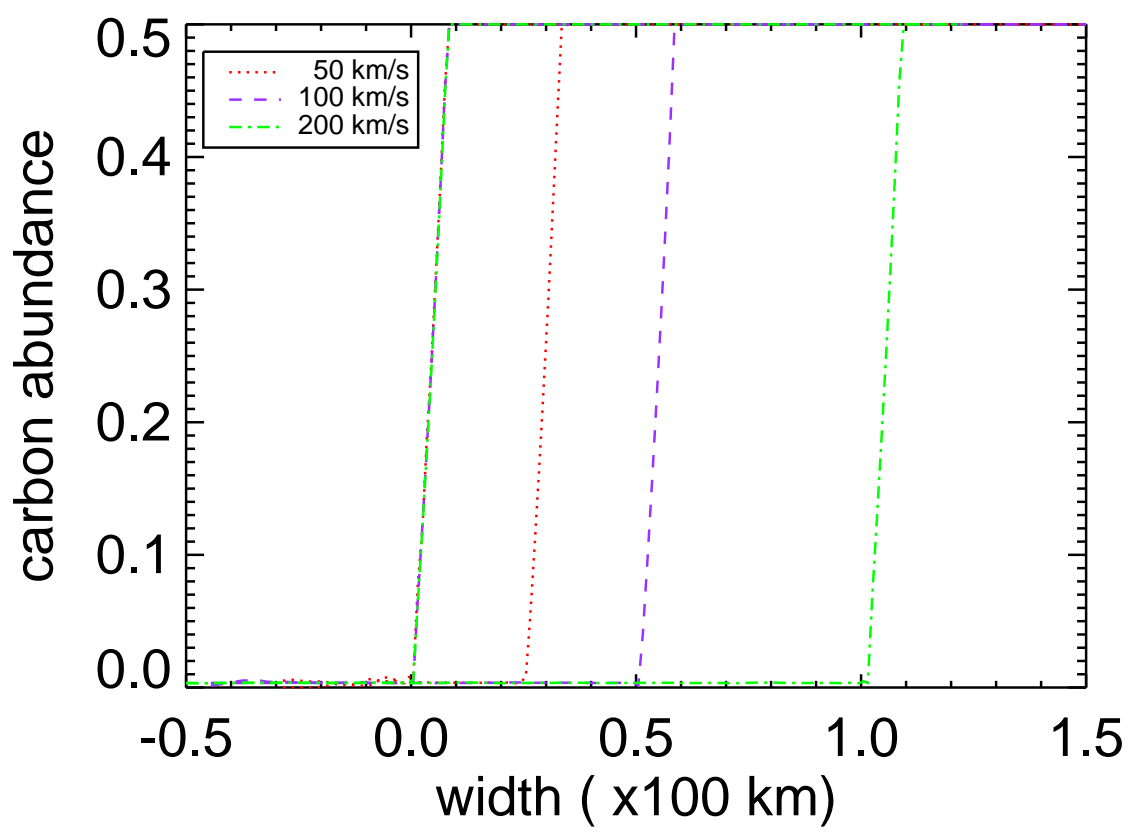

Fig. 2.- 1D laminar flame propagation for flame speeds of 50, 100 and $200 \mathrm{~km} \mathrm{~s}^{-1}$. Flame fronts at $0.3 \mathrm{~s}$ are put at the same location, and flame fronts for different prescribed speeds at $0.8 \mathrm{~s}$ are shown. 

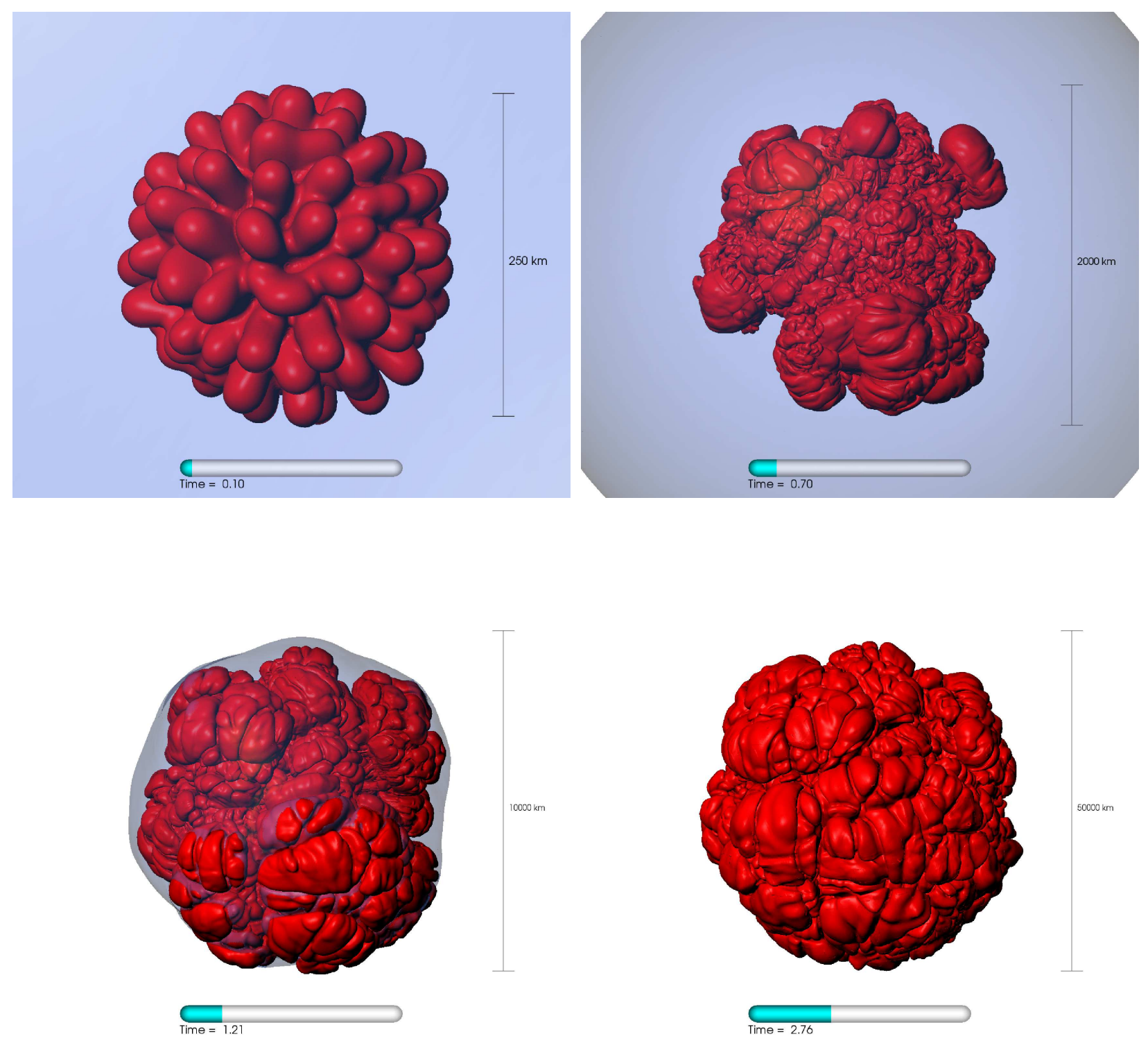

Fig. 3.- Contour plots of the ash-fuel interfaces showing the development of the thermonuclear deflagration in the carbon-oxygen white dwarf in the simulation with flame speed parameter $100 \mathrm{~km} / \mathrm{s}$, model A100. Star time and length scale are shown on each plot. Red color indicates the interface, and light blue color indicates the star surface where density equals $10^{6} \mathrm{~g} \mathrm{~cm}^{-3}$. 


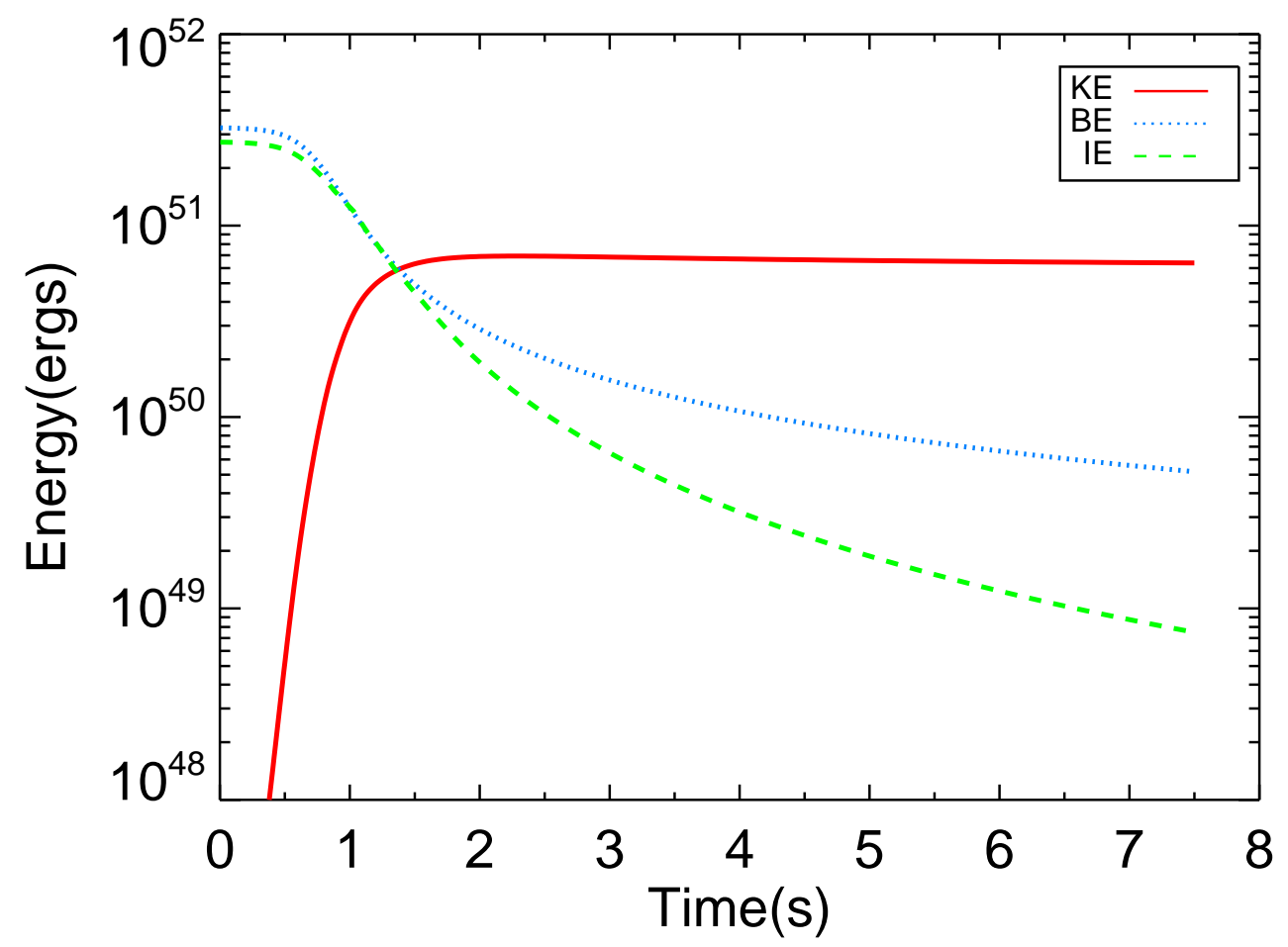

Fig. 4.- Kinetic energy, binding energy and internal energy evolution for model A100. 

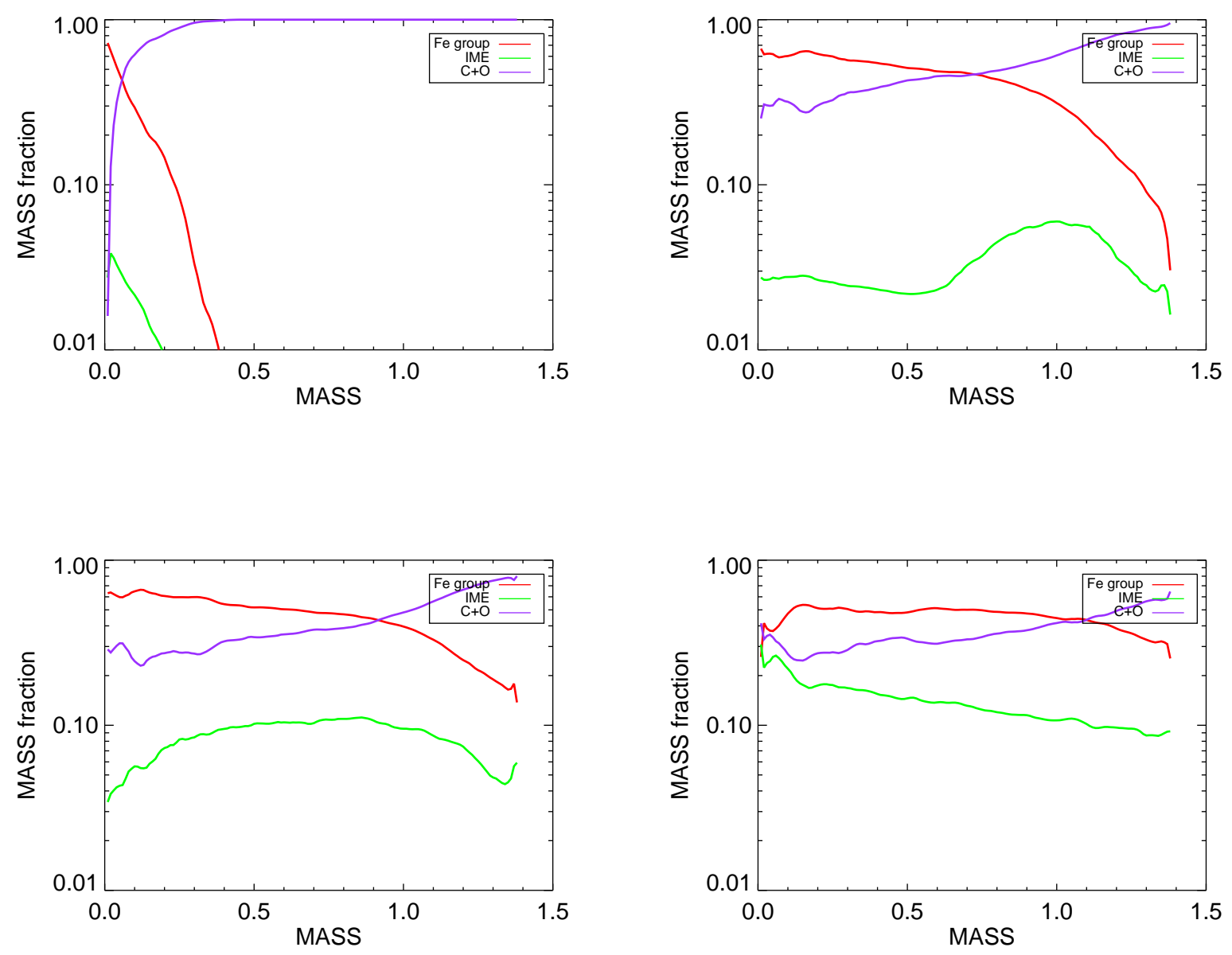

Fig. 5.- Mass fraction of iron, IME and unburned material at $0.5 \mathrm{~s}, 1 \mathrm{~s}, 1.2 \mathrm{~s}$ and $1.7 \mathrm{~s}$ for model A100. 

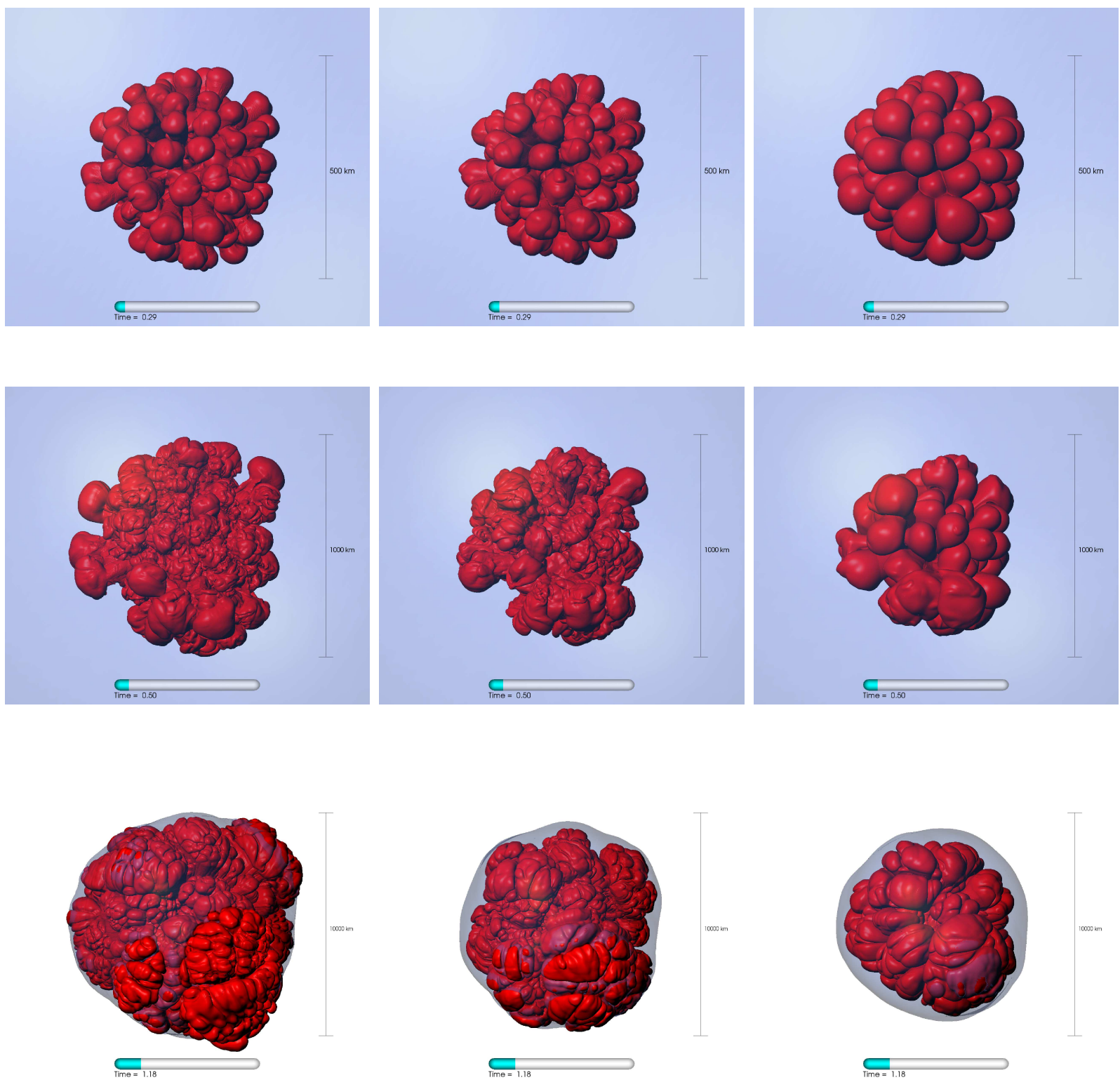

Fig. 6.- Contour plots of the burning interfaces for 3 different prescribed flame speed models, A50, A100 and A200 (from left to right) at the star time of $0.3 \mathrm{~s}, 0.5 \mathrm{~s}$ and $1.2 \mathrm{~s}$ (from top to bottom). 


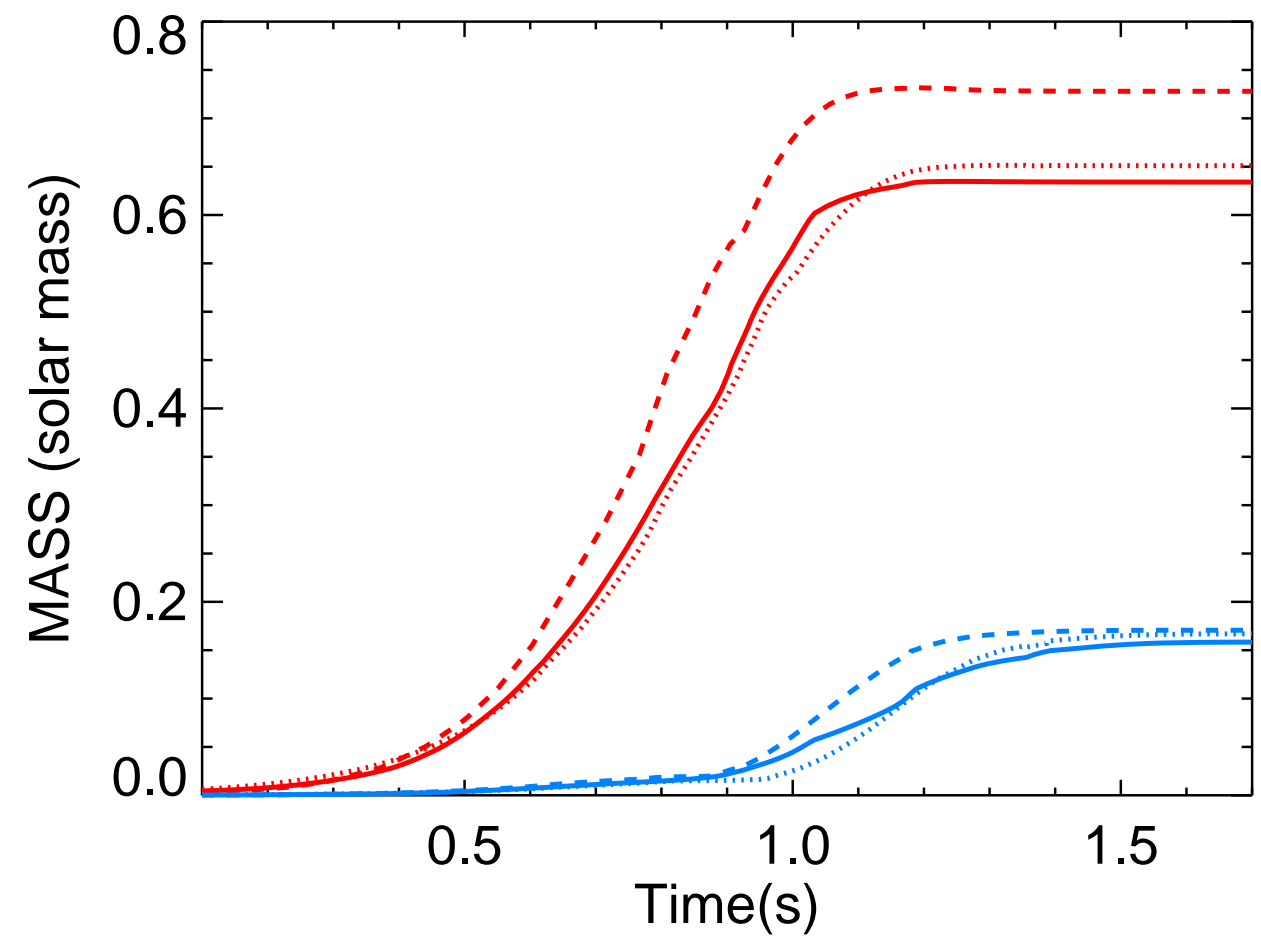

Fig. 7. - Mass of produced iron group elements (red) and IME (blue) with time for 3 different prescribed flame speed models. The solid lines correspond to model A100, the dashed lines to model A50, and the dotted lines to A200. 

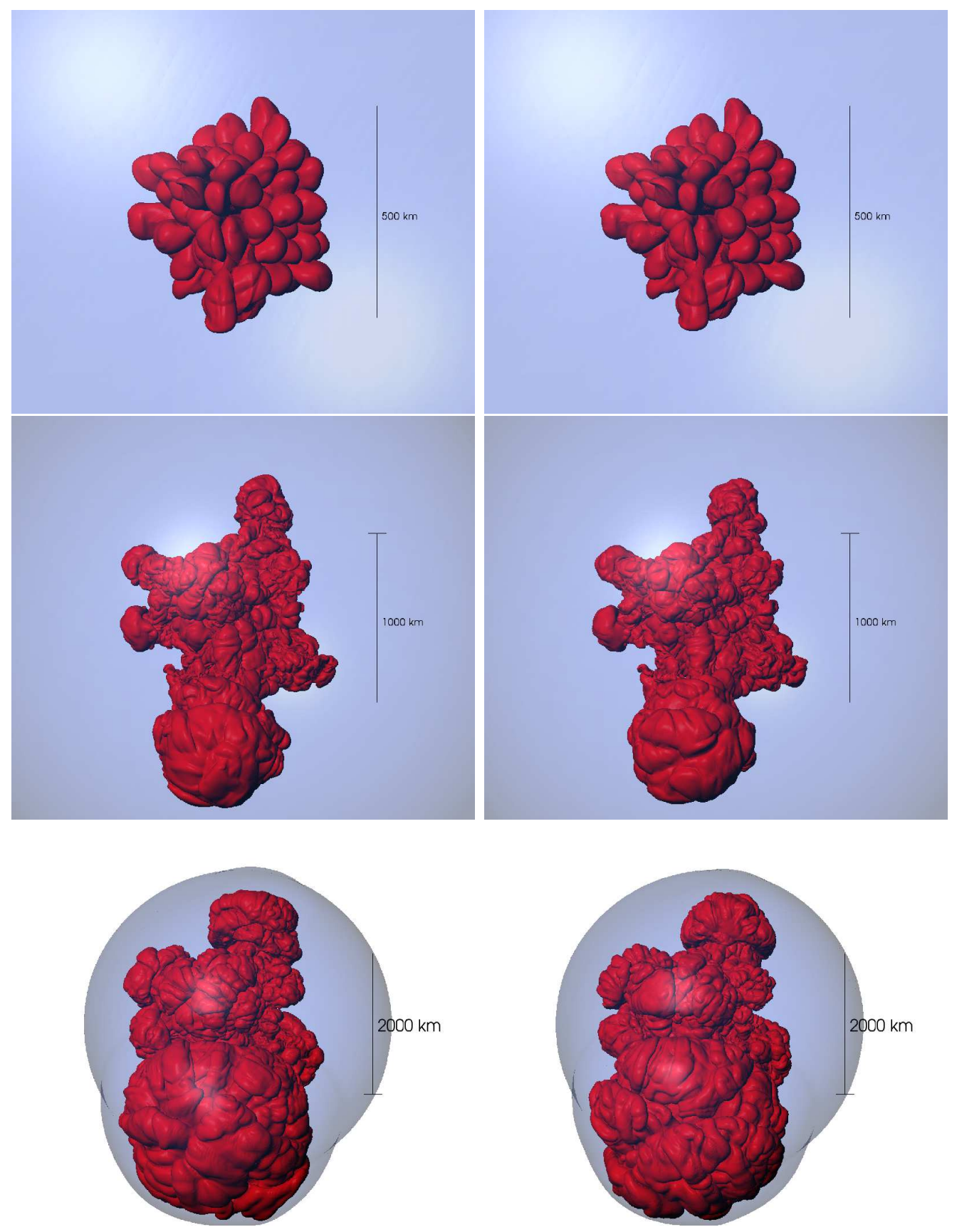

Fig. 8.- Similar plots to Figure 6, but for the B series of models. The left column is model $\mathrm{B} 100$ and the right column is model BT; snapshots are (from top to bottom) at $0.3 \mathrm{~s}, 0.5 \mathrm{~s}$, and $0.7 \mathrm{~s}$. 


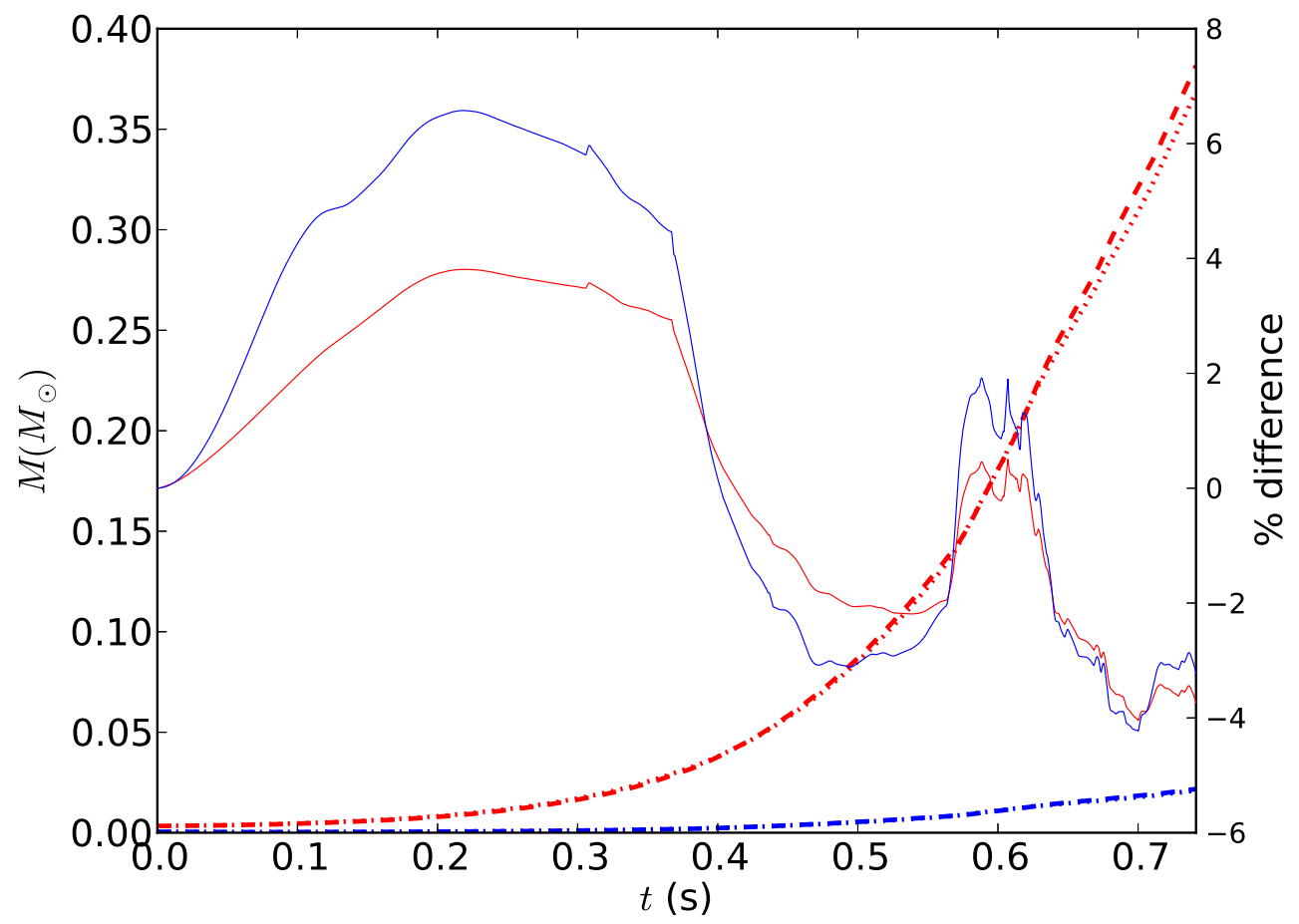

Fig. 9.- Total mass production of iron group elements (red) and IME (blue) for models B100 (dotted) and BT (dashed). The thin lines show the percent difference between the B100 and BT models for each element group; a positive (negative) percent difference indicates that model B100 has produced more (less) material. 


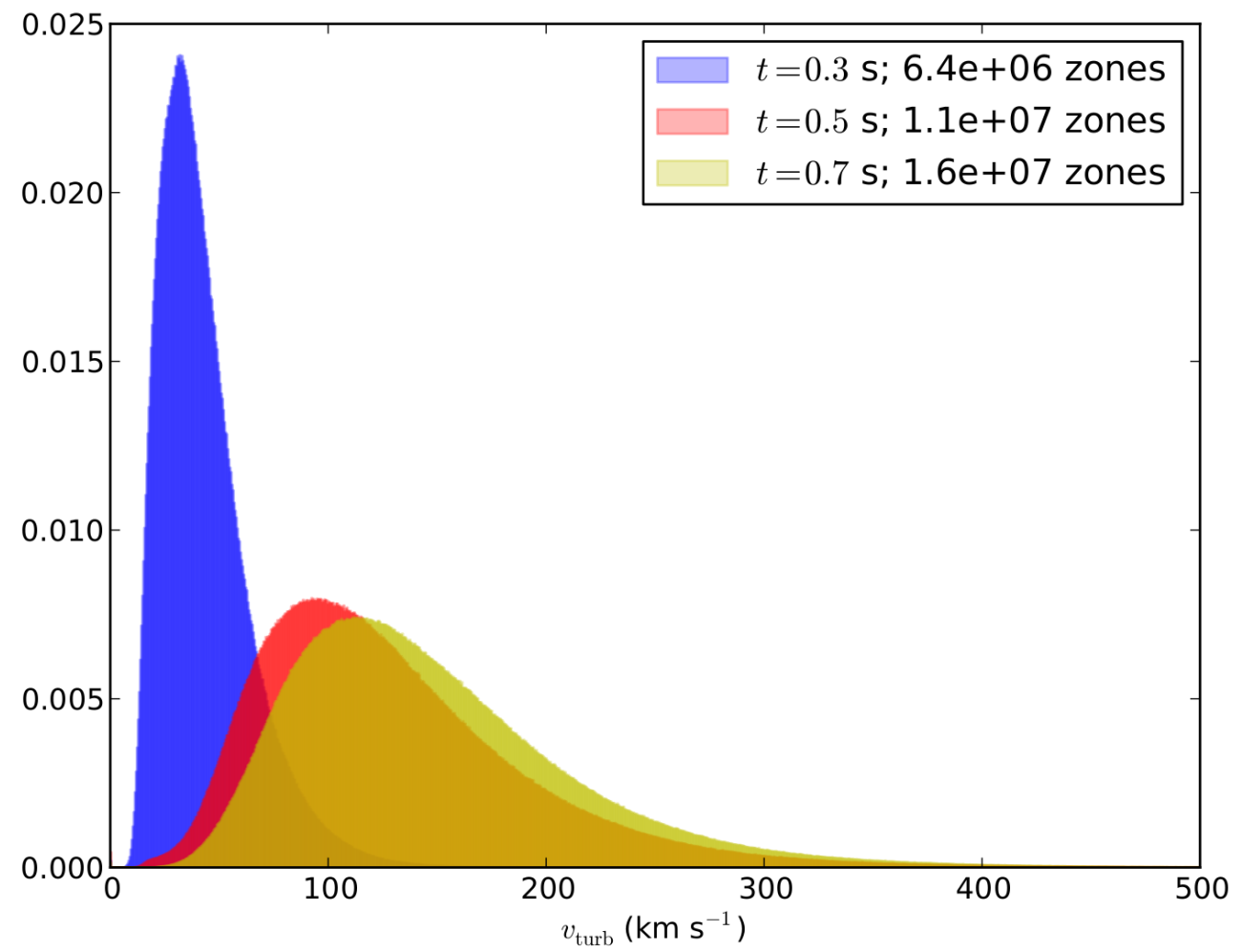

Fig. 10.- PDF of turbulent velocity inside the flame for model BT at various times. Early on, the majority of the zones within the flame experience a turbulent intensity that is slower than the laminar speed, and therefore the flame propagates laminarly. As the system evolves, the distribution widens and peaks towards larger flame speeds. 

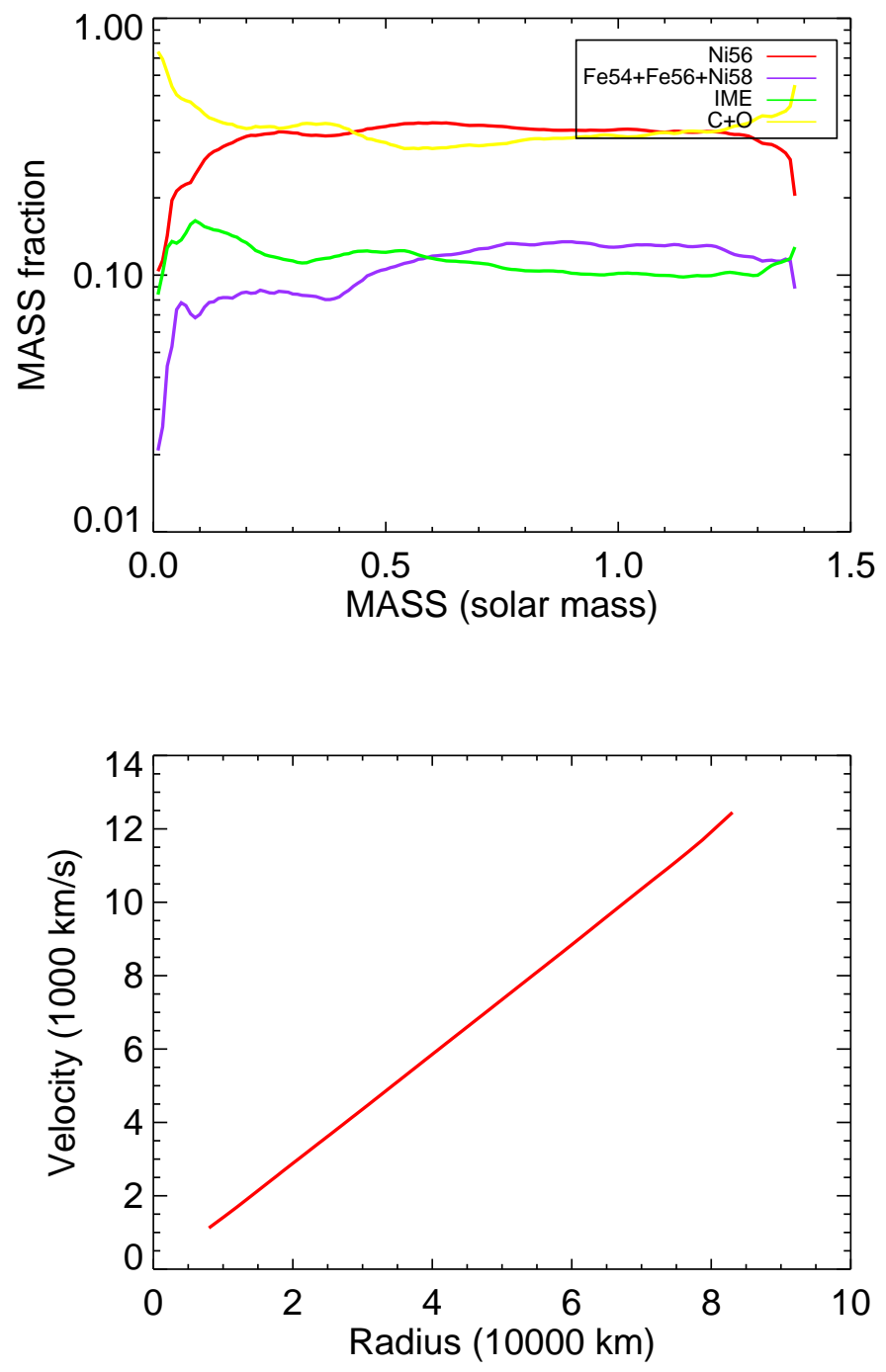

Fig. 11. - Mass fraction of ${ }^{56} \mathrm{Ni}$, other iron group elements, IME and unburned $\mathrm{C}+\mathrm{O}$ at $7.5 \mathrm{~s}$ for model A100. The plot of velocity versus radius shows the supernova ejecta is in homologous expansion. 


\section{REFERENCES}

Almgren, A. S., Beckner, V. E., Bell, J. B., et al. 2010, ApJ, 715, 1221

Benetti, S., et al. 2005, ApJ, 623, 1011

Branch, D., Doggett, J. B., Nomoto, K., \& Thielemann, F. K. 1985, ApJ, 294, 619

Branch D., \& Khokhlov, A. M. 1995, Phys. Rep., 256, 53

Branch, D., et al. 2003, AJ, 126, 1489

Ciaraldi-Schoolmann, F., Schmidt, W., Niemeyer, J. C., Röpke, F. K., \& Hillebrandt, W. 2009, ApJ, 696, 1491

Damköhler, G. 1940, Z. Elektrchem, 46, 601

Davies, R. M., \& Taylor, G. 1950, Proc. R. Soc. London A, 200, 375

Filippenko, A. V. 1997, ARA\&A, 35, 309

Gamezo, V. N., Khokhlov, A. M., Oran, E. S., Chtchelkanova, A. Y., \& Rosenberg, R. O. 2003, Science, 299, 77

Gamezo, V. N., Khokhlov, A. M., \& Oran, E. S. 2005, ApJ, 623, 337

Garcia-Senz, D., \& Woosley, S. E. 1995, ApJ, 454, 895

García-Senz, D., \& Bravo, E. 2005, A\&A, 430, 585

Gilfanov, M., \& Bogdán, Á. 2010, Nature, 463, 924

Hachisu, I., Kato, M., \& Nomoto, K. 2010, ApJ, 724, L212

Hartmann, D., Woosley, S. E., \& El Eid, M. F. 1985, ApJ, 297, 837

Heger, A., Woosley, S. E., Martinez-Pinedo, G., \& Langanke, K. 2001, ApJ, 560, 307

Hillebrandt, W., \& Niemeyer J. 2000, ARAA, 38, 191b

Höflich, P., \& Khokhlov, A. 1996, ApJ, 457, 500

Höflich, P., \& Stein, J. 2002, ApJ, 568, 779

Howell, D. A., Sullivan, M., Nugent, P. E., et al. 2006, Nature, 443, 308 
Hoyle, F., \& Fowler, W. A. 1960, ApJ, 132, 565

Jackson, A. P., Calder, A. C., Townsley, D. M., Chamulak, D. A., Brown, E. F. \& Timmes, F. X. 2010, ApJ, 720, 99

Kasen, D., Röpke, F., \& Woosley, S. E. 2009, Nature, 460, 869

Khokhlov, A. M. 1995, ApJ, 449, 695

Khokhlov, A. M. 1996, Combust. Flame, 105, 28

Khokhlov, A. M., Oran, E. S., \& Wheeler, J. C. 1997, ApJ, 478, 678

Kromer, M., Fink, M., Stanishev, V., Taubenberger, S., Ciaraldi-Schoolman, F., Pakmor, R., Röpke, F. K., Ruiter, A. J., Seitenzahl, I. R., Sim, S. A., Blanc, G., Elias-Rosa, N., \& Hillebrandt, W. 2013, MNRAS, 429, 2287

Krueger, B. K., Jackson, A. P., Calder, A. C., Townsley, D. M., Brown, E. F. \& Timmes, F. X. 2012, ApJ, 757, 175

Kuhlen, M., Woosley, S. E., \& Glatzmaier, G. A. 2006, ApJ, 640, 407

Landau, L., \& Lifshitz, F. M. 1959, Course in Theoretical Physics, Vol. 6. Fluid Mechanics (Oxford:Pergammon)

Langanke, K. \& Martinez-Pinedo, G. 2000, Nucl. Phys. A, 673, 481

Marion, G. H., Höflich, P., Vacca, W. D. ,\& Wheeler, J. C. 2003, ApJ, 591, 316

Mazzali, P. A., Röpke, F. K., Benetti, S., \& Hillebrandt, W. 2007, Science, 315, 825

Niemeyer, J. C., \& Woosley, S. E. 1997, ApJ, 475, 740

Nomoto, K., Thielemann, F. K., \& Yokoi, K. 1984, ApJ, 286, 644

Nonaka, A., Aspden, A. J., Zingale, M., et al. 2012, ApJ, 745, 73

Osher, S., \& Sethian, J. A. 1988, J. Comp. Phys., 79, 12

Pakmor, R., Hachinger, S., Röpke, F. K., \& Hillebrandt, W. 2011, A\&A, 528, A117

Parrent, J. T., et al. 2011, ApJ, 732, 30

Perets, H. B., Gal-Yam, A., Mazzali, P. A., et al. 2010, Nature, 465, 322

Plewa, T., Calder, A. C., \& Lamb, D. Q. 2004, ApJ, 612, L37 
Reinecke, M., Hillebrandt, W., Niemeyer, J. C., Klein, R., \& Gröbl, A. 1999, A\&A, 347, 724

Reinecke, M., Hillebrandt, W., \& Niemeyer, J. C. 2002, A\&A, 391, 1167

Röpke, F. K., \& Hillebrandt, W. 2005, A\&A, 431, 635

Röpke, F. K., Hillebrandt, W., Niemeyer, J. C., \& Woosley, S. E. 2006, A\&A, 448, 1

Röpke, F. K. 2007, ApJ, 668, 1103

Röpke, F. K., Hillebrandt, W., Schmidt, W., et al. 2007a, ApJ, 668, 1132

Röpke, F. K., Woosley, S. E., \& Hillebrandt, W. 2007b, ApJ, 660, 1344

Schmidt, W., Niemeyer, J. C., \& Hillebrandt, W. 2006a, A\&A, 450, 265

Schmidt, W., Niemeyer, J. C., Hillebrandt, W., Röpke, F. K. 2006b, A\&A, 450, 283

Schmidt, W., \& Niemeyer, J. C. 2006, A\&A, 446, 627

Sharp, D. H., 1984, Physica D:Nonlinear Phenomena, 12, 3

Stritzinger, M., Leibundgut, B., Walch, S., \& Contardo, G. 2006, A\&A, 450, 241

Timmes, F. X., \& Woosley, S. E. 1992, ApJ, 396, 649

Timmes, F. X., \& Swesty, F. D., 2000, ApJS, 126, 501

Townsley, D. M., Calder, A. C., Asida, S. M., et al. 2007, ApJ, 668, 1118

Voss, R., \& Nelemans, G. 2008, Nature, 451, 802

Weaver, T. A., Woosley, S. E., \& Zimmerman, G. B. 1978, ApJ, 225, 1021

Woosley, S. E., Heger, A., \& Weaver, T. A. 2002, Reviews of Modern Physics, 74, 1015

Woosley, S. E., Kasen, D., Blinnikov, S., \& Sorokina, E. 2007, ApJ, 662, 487

Woosley, S. E. 2007, ApJ, 668, 1109

Woosley, S. E., Kerstein, A. R., Sankaran, V., Aspden, A. J., \& Röpke, F. K. 2009, ApJ, 704,255

Woosley, S. E., \& Kasen, D. 2011, ApJ, 734, 38

Woosley, S. E., Kerstein, A. R., \& Aspden, A. J. 2011, ApJ, 734, 37 
Yoon, S.-C., Podsiadlowski, P., \& Rosswog, S. 2007, MNRAS, 380, 933

Zingale, M., Woosley, S. E., Rendleman, C. A., Day, M. S., \& Bell, J. B. 2005, ApJ, 632, 1021

Zingale, M., \& Dursi, L. J. 2007, ApJ, 656, 333

Zingale, M., Almgren, A. S., Bell, J. B., Nonaka, A., \& Woosley, S. E. 2009, ApJ, 704, 196

Zingale, M., Nonaka, A., Almgren, A. S., et al. 2011, ApJ, 740, 8 